\title{
Single-cell Atlas Unveils Cellular Heterogeneity and Novel Markers in Human Neonatal and Adult Intervertebral Discs
}

Wensen Jiang ${ }^{1,2}$, Juliane D. Glaeser ${ }^{1,2,5,6}$, Khosrowdad Salehi ${ }^{1,2}$, Giselle Kaneda ${ }^{1,2}$, Pranav Mathkar ${ }^{2}$, Anton Wagner ${ }^{2}$, Ritchie $\mathrm{Ho}^{2,3,4}$, Dmitriy Sheyn ${ }^{1,2,5,6,7 *}$

${ }^{1}$ Orthopaedic Stem Cell Research Laboratory, Cedars-Sinai Medical Center, Los Angeles, California, USA, 90048

${ }^{2}$ Board of Governors Regenerative Medicine Institute, Cedars-Sinai Medical Center, Los Angeles, California, USA, 90048

${ }^{3}$ Center for Neural Sciences and Medicine, Cedars-Sinai Medical Center, Los Angeles, California, USA, 90048

${ }^{4}$ Department of Neurology, Cedars-Sinai Medical Center, Los Angeles, California, USA, 90048

${ }^{5}$ Department of Orthopedics, Cedars-Sinai Medical Center, Los Angeles, California, USA, 90048

${ }^{6}$ Department of Surgery, Cedars-Sinai Medical Center, Los Angeles, California, USA, 90048

${ }^{7}$ Department of Biomedical Sciences, Cedars-Sinai Medical Center, Los Angeles, California, USA, 90048

${ }^{*}$ Corresponding author

Correspondence:

Dmitriy Sheyn, PhD

Board of Governors Regenerative Medicine Institute

Department of Orthopedics

Department of Surgery

Department of Biomedical Sciences

Cedars-Sinai Medical Center

AHSP A8308, 8700 Beverly Boulevard

Los Angeles, CA 90048

Email: dmitriy.sheyn@csmc.edu 
SUMMARY

The origin, composition, distribution, and function of cells in the human intervertebral disc (IVD) has not been fully understood. Here, cell atlases of both human neonatal and adult IVDs have been generated and further assessed by gene ontology pathway enrichment, pseudo-time trajectory, histology, and immunofluorescence. Comparison of cell atlases revealed the presence of two sub-populations of notochordal cells (NCs) and their associated markers in both the neonatal and adult IVDs. Developmental trajectories predicted 7 different cell states that describe the developmental process from neonatal to adult cells in IVD and analyzed the NC's role in the IVD development. A high heterogeneity and gradual transition of annulus fibrosus cells (AFCs) in the neonatal IVD was detected and their potential relevance in IVD development assessed. Collectively, comparing single-cell atlases between neonatal and adult IVDs delineates the landscape of IVD cell biology and may help discovering novel therapeutic targets for IVD degeneration.

\section{KEYWORDS}

single-cell RNA sequencing; human intervertebral disc; intervertebral disc development; notochordal cells; nucleus pulposus cells; annulus fibrosus cells 


\section{INTRODUCTION}

At least $30 \%$ of total adult population is suffering from lower back pain, which originates in intervertebral disc (IVD) degeneration (Andersson, 1999; de Schepper et al., 2010; Frank et al., 1996; Macfarlane et al., 1999) To date, most treatments of IVD degeneration are limited to invasive surgical interventions, such as disc replacement and spinal fusion, or pain management that is not addressing the underlying cause of IVD degeneration (Knezevic et al., 2017).

The IVD is a shock-absorbing structure that connects two adjacent vertebras and enables spine movement (Buckley et al., 2018; Vergroesen et al., 2015). The human IVD cell composition is highly heterogenous; nucleus pulposus (NP) is the inner core and the annulus fibrosus (AF) is the outer region that is confined by two endplates sandwiching the disc (Sun et al., 2020). It is widely accepted the embryonic notochord develops into the NP and that notochordal cells (NCs) disappear within the first decade of life (Séguin et al., 2018). However, the NC remnants or dormant NC can be found in the adult human IVD (McCann and Séguin, 2016; Wang et al., 2008). Additional study showed that the NCs or NC-like NP cells (NPCs) in human persist throughout life (Risbud and Shapiro, 2011). The eventual fate of human NCs is not conclusive to date, but understanding its development has considerable potential to benefit cell therapies of IVD degeneration. Animals that keep the NCs in adulthood like cats and pigs do not to exhibit disc degeneration (Hunter et al., 2003; Sheyn et al., 2019). NCs hold great potential to rejuvenate a degenerated human IVD and this potential has already been demonstrated in a mini pig model (Sheyn et al., 2019).

Recent advances in single-cell RNA sequencing (scRNA-seq) allow for creation of cell atlases that unveil rare sub-populations, delineate cellular heterogeneity, identify new markers, and predict developmental trajectories (Ho et al., 2021; Kelly et al., 2020; Mathys et al., 2019; Setty et al., 2019; Stark et al., 2019). Unraveling the single-cell atlas and transcriptomes of the human IVD at different ages will largely extend our understanding of its cell biology and development. To date, its heterogeneity, particularly in the early development, has not been sufficiently shown due 
to the limitation of traditional bulk-sequencing (Fernandes et al., 2020). Previously, multiple bulk proteomics studies delineated the general cell heterogeneity of human IVD (Rodrigues-Pinto et al., 2018; Tam et al., 2020b) and made comparison at different developmental stages (Tam et al., 2020b). Recent studies of single-cell atlases in non-human vertebrates resolved the cellular heterogeneity in bovine caudal IVD (Panebianco et al., 2021) and found stem cells in rat IVD (Wang et al., 2021). Another study provided single-cell analysis of human adult IVD that identified the NPC and AFC populations (Fernandes et al., 2020). A recent study identified chondrocytes as dominating cell population in human IVD and found small NC sub-populations (Gan et al., 2021). Yet no study has investigated cell atlases from neonatal human IVD and thus elaborated the role of NCs in development and hemostasis at a single-cell resolution.

Here, we analyze a human IVD single-cell atlas with direct head-to-head comparison between neonatal and adult human subjects to reveal the identities of all IVD populations and subpopulations of both developmental stages, discover rare cell populations and novel markers thereof, and predict the developmental trajectories from neonatal to adult IVD. We isolated cells from neonatal and adult IVDs postmortem, and analyzed them with scRNA-seq. An in-depth gene ontology term enrichment and pathway analysis was performed and developmental trajectories from neonatal to adult IVD were predicted. We further validated our findings with histology and immunofluorescence.

\section{RESULTS}

Human Neonatal and Adult IVD Cells are Comprised of 14 Distinct Sub-populations

Histological analysis of neonatal IVD tissue demonstrated two distinct tissue regions. Region A contained aggregated cells and loose extracellular matrix (ECM) structures and region $B$ contained sparsely distributed cells and dense ECM tissue (Fig. 1a). Vessels and red blood cells were clearly identified in region A (Fig. 1a). In contrast, the adult IVD did not exhibit different 
regions. The uniform and homogenous tissue contained sparsely distributed cells whose cytoplasm was greatly larger and cell density lower than in neonatal IVD (Fig. 1b).

Dimensional reduction based on the unsupervised UMAP method sorted all cells into 15 clusters that could be classified as 15 cell sub-populations residing in both neonatal (Fig. 1c) and adult IVDs (Fig 1d). The expression levels of classical IVD markers in each cluster were shown as dot plot for neonatal IVD (Fig. 1c) and adult IVD (Fig. 1d). The selection of classical IVD-relevant markers includes NC markers MAP1B (Rodrigues-Pinto et al., 2018), SOX4 (Bhattaram et al., 2010), SOX17 (D'Amour et al., 2005; Sheyn et al., 2019), ITGA6 and BASP1 (Sheyn et al., 2019), NPC markers ACAN (Fernandes et al., 2020; Risbud et al., 2015; Tam et al., 2020a), COL2A1 (Fernandes et al., 2020; Risbud et al., 2015; Tam et al., 2020a) and SOX9 (Sheyn et al., 2019), AFC markers COL1A1 (Tam et al., 2020b; van den Akker et al., 2017), CALR (Tam et al., 2020b) and HSPA6 (Takao and Iwaki, 2002), as well as other previously shown markers like MEG3 for non-mesenchymal cells (Chen et al., 2017), CD44 (Schumann et al., 2015) and CD14 (ZieglerHeitbrock and Ulevitch, 1993) for monocytes, and HBB (Talamo et al., 2003) and HBA1 (Pandey and Rizvi, 2011) for red blood cells (RBC, Fig. 1e-f). Based on these markers we annotated each cluster (Fig. 1g). The distribution of cells from neonatal vs. adult IVD in each annotated subpopulation, normalized by total cells of their respective age, shows that neonatal IVD disproportionally contributed to NC1, NPC3-4, (inner) iAFC and (outer) oAFC1 clusters (Fig. 1h). Adult IVD disproportionally contributed to the NC2, NC/NPC, NPC2, and RBC2 clusters. The contribution to rest of the sub-populations was more or less evenly distributed.

Human Neonatal and Adult Intervertebral Disc Contain Two Distinct Notochordal Cell SubPopulations

Cluster 1 and 2 in the neonatal IVD were identified as NC sub-populations (Fig. 1c-h) based on their expression of NC markers, MAP1B (Rodrigues-Pinto et al., 2018) and SOX4 (Bhattaram et al., 2010), and have been assigned to NC1 and NC2 (Fig. 2a). NC1 and NC2 were found to be distinct from each other: NC1 expressed SOX17 and ITGA6, while NC2 expressed CD44 (Fig. 2a). The NC sub-populations were also found in adult IVD (Fig. 2a). NC2 showed overwhelmingly higher cell counts than NC1 in adult (Fig. 1h and 2a). Immunostaining of the NC markers, MAP1B 
and SOX17, confirmed their expression in both neonatal and adult IVDs (Fig. 2b) on the protein level. These proteins showed a higher overall expression in neonatal than in adult IVD. We also observed the NC2 expressed immune-relevant marker CD44 (Fig. 2a) and confirmed the presence of CD44 proteins in both ages (Fig. 2b).

Pathway networks were further created based on the list of all enriched genes for NC1 and NC2 (Fig. S1a, b), that is, 860 genes for NC1 and 583 genes for NC2. Our results show NC1 preferentially demonstrated the activities of cancer-related pathway STAT1 and the deactivation of Brachyury (TBXT), Microphthalmia-associated Transcription Factor (MITF), and SWI/SNF Related, Matrix Associated, Actin Dependent Regulator of Chromatin (SMARCD3), which is further associated with the deactivation of connective tissue cell pathways and the collagenrelevant Glycoprotein VI (GP6) signaling pathway, and the deactivation of connective tissue activities (Fig. S2b). MITF was previously shown to be associated with osteoclast activities (Lu et al., 2010) and GP6 was shown to induce collagen deposition (Stegner et al., 2014). NC2 preferentially demonstrated stem cell pluripotency factor FGF2 (Fig. S2a) which led to the organization of cytoplasm and organization of cytoskeleton.

$A K R 1 C 1, A P O E$, and FABP4 are Novel Markers for Notochordal Cells in Human Intervertebral Disc

We selected several NC markers from top enriched genes that are shown in Fig. 2c. Specifically, FABP4 is a strong marker for NC1 in both neonatal and adult IVD, AKR1C1 is the NC2 marker for neonatal IVD, and $A P O E$ is a relatively weak marker but can be identified in the entire NC population (Fig. 2c). Interestingly, AKR1C1 is the only marker common to the entire NC population in adult IVD (Fig. 2c). We compared the expression levels of FABP4, AKR1C1, and APOE in neonatal and adult IVDs due to their possible age-dependent specificity (Fig. 3a). FABP4 is only specific to neonatal NC1. AKR1C1 lost certain specificity in adult IVD (Fig. 3a) but is still specific to the entire NC population and NC2 (Fig. 2c). The immunostaining of novel NC markers, AKR1C, FABP4, and $A P O E$, confirmed their expression in both neonatal and adult IVDs. All markers show overlap with cell nucleus besides $A K R 1 C$ that appears in the cytoplasm. 
We further investigated the role of the new markers in the regulatory networks and biofunctions. We found that FABP4 plays a critical role in the VEGF-regulated network in neonatal IVD, in which the upregulation of VEGF leads to the activation of NC markers FABP4 and ITGA6, inflammatory factor CXCL8, and downregulate ACAN (Fig. 3c). In adult IVD, however, FABP4 is activated by P38 MAPK regulator (Fig. 3c). AKR1C1 and AKR1C2 play a critical role in the TGFB1 cascade in the neonatal NC2 sub-population (Fig. 3d) This network seems to be highly relevant to NCs as it also leads to the activation of classical NC markers MAP1B and immune-relevant CD44 (Fig. 3d). The activation of $A K R 1 C 1$ is associated with multiple biological functions, as shown in Fig. 3e. Specifically, the upregulation of $A K R 1 C 1 / A K R 1 C 2$ activates multiple tumor and cancer-related functions (Fig. 3e). Lastly, we noticed the novel APOE marker was in the IL1 cascade, which also regulates $A K R 1 C 1 / A K R 1 C 2$. (Fig. S1C).

Nucleus Pulposus Cell Populations Contain Four Similar Sub-populations with Different Biological Functions in Neonatal Versus Adult Intervertebral Disc

NPCs expressing their classical markers, ACAN (Fernandes et al., 2020; Risbud et al., 2015; Tam et al., 2020a), COL2A1 (Fernandes et al., 2020; Risbud et al., 2015; Tam et al., 2020a), and SOX9 (Sheyn et al., 2019) were detected in both neonatal IVD (Fig. 4a) and adult IVD (Fig. 4b). The entire NPC population contained four different sub-populations identified by their specific markers: NPC1 identified by OGN, NPC2 identified by MMP13, NPC3 identified by COL9A1, and NPC4 identified by DPT. The clusters 4-7 (Fig. 1c-d) thus have been designated as NPC1-4 respectively. Cluster 3 (Fig. 1c-d) partly expresses NC markers (SOX4+ but no MAP1B) and partly expresses NPC markers (COL2A1+ but no ACAN and SOX9), and thus it has been designated as NC/NPC. It may represent a transition state of cells from NC to NPC or a reservoir of NP progenitors. The expression of the NPC markers is shown in violin plots (Fig. 4a-b). However, four NPC sub-populations from the same age are barely distinguishable (Fig. 4a, b). The subpopulation markers are not very specific as the gene expression distributed on the UMAP did not always match the clustering on the UMAP (Fig. 4a, b). The four NPC sub-populations may have similar transcriptomic profiles. The AFC marker levels were used as controls (Fig. 4a, b) and their expression was found very low among all NPCs in both the neonatal and adult IVD, which 
confirmed the NPC clusters are not overlapping with AFCs. Only NPC4 in neonatal IVD expressed AFC marker COL1A1 (Fig. 4a).

NPCs are critical to the hemostasis and deposition of ECM (Frapin et al., 2019). We observed that the age greatly affected the critical ECM proteins (Fig. 4c). Figure 4c shows the abundant presence of collagen type 1 (COL1A1) in the extracellular space and aggrecan in the cytoplasm in the neonatal IVD. The signal of intracellular COL1A1 appears to be weak in neonatal IVD (Fig. 4c). Figure 4c shows the opposite trend for adult IVD: the staining of COL1A1 in the extracellular space of neonatal IVD is negligible, but the staining in the cytoplasm is strong; the staining of aggrecan in the extracellular space is strong, but weak in the cytoplasm (Fig. 4c).

Notochordal Cell and Nucleus Pulposus Cell Populations Exhibit Different Gene Ontology Enrichments, Pathways, and Regulators

NC and NPC populations were compared in both neonatal and adult IVDs (Fig. 4d-f). In this analysis, NC1-2 were combined as NCs, and NPC1-4 were combined as NPCs (Fig. 4d). NC populations were preferentially enriched to cancer and tumor activities, cell metabolism, cellular homeostasis, angiogenesis, and cell adhesion and spreading, whereas NPC populations were preferentially enriched to cytoskeletal tissue formation, the development of cytoplasm, wounding healing and ECM development (Fig. 4d). We also showed top-scored key regulatory networks in neonatal NCs and adult NPCs in Fig. S1d. Several inflammatory and tumorigenic factors and pathways (IL1B, VEGF, HGF) were upregulated in NC (Fig. S1d). In NPCs, upregulation of the osteogenic factor BMP2 (Cai et al., 2021), osteoclast regulator MITF (Lu et al., 2010), DNAbinding transcription factor-related BCL6, NPC and skeletal development marker SOX9 (Bi et al., 1999; Sheyn et al., 2019; Zhou et al., 2006) were detected (Fig. S1d). This trend seems to be age-independent except for BCL6, which was not detected in the adult IVD (Fig. S1d). Different cell types (NC vs. NPC) demonstrated hugely distinct transcriptomes, but the differences between the ages (neonatal vs. adult) was much smaller, as summarized in the Circos plot (Fig. 4e), where the overlap of expressed genes mostly occurred among the same cell types. The summary of enriched functional terms is presented in Fig. $4 \mathrm{f}$. 
Annulus Fibrosus Cells in Neonatal IVD are Highly Heterogenous, and their Expression of Collagen-Relevant Genes is Location-Dependent

COL1A1 is the most common ECM protein secreted by AFCs (Tam et al., 2020b; van den Akker et al., 2017). CALR is a marker for the outer AF (Tam et al., 2020b) and heat shock proteins (HSPS, e.g. HSPA6, HSPA1A, etc.) are markers for the most outer layer of the AF (Takao and Iwaki, 2002). Thus, the COL1A1, CALR, and HSPA6 were used to identify AFC populations (Fig. $5 a, b)$. Cluster 8 (Fig. 1c-d) has been classified as the inner AFC sub-population (iAFC), due to its expression of COL1A1 (Fig. 5a, b). The clusters 9-11 (Fig. 1c-d) have been assigned to the outer AFC sub-population (oAFC1-3) respectively, due to their expression of CALR (cluster 9,10 ) and HSPA6 (cluster 11, Fig. 5a, b). We detected a high degree of heterogeneity among neonatal AFC populations (Fig. 5c). We identified LGALS1, HES1, HERPUD1, and DNAJB1 as AFC subpopulation markers for iAFC and oAFC1-3. Moreover, several types of collagens were also detected in neonatal IVD, specifically COL1A1, COL3A1, COL5A1, COL5A2, COL6A3, COL12A1 (Fig. 5d). Strikingly, their expression showed a decreasing trend assuming the location of subtypes follows the order of iAFC, OAFC1, OAFC2 and oAFC3 from inner core to the outer region (Fig. 5d). A similar trend was observed in the enriched biofunctions and key pathway analyses (Fig. 5e) across the neonatal iAFC and oAFC populations. As part of the pathways listed in Fig. $5 e$, we show the mechanistic scheme of Unfolded Protein Response (UPR) signaling pathway and the relevant gene expression in oAFC2 in Fig. S2. The adult IVD did not exhibit similar trend of collagen-relevant gene expression (Fig. S3). The illustration in Fig. $5 f$ shows the postulated structure transition from neonatal to adult IVD based on these spatially dependent gene expression patterns.

Resolved Single-cell Atlas for Human Neonatal and Adult IVD with Clusters Assigned to Subpopulations

We have assigned cluster 12 into non-mesenchymal cells (non-MC) due to their MEG3 expression, a marker for non-mesenchymal, tumor-suppressing cells (Chen et al., 2017). Cluster 13 is a satellite cell population on the UMAP (Fig. 1c-d). It shared the CD44 expression as NC2 
but lacked all other NC markers (Fig. 1c, d). Thus, we have assigned cluster 13 to immune-like cells (IC) due to its expression of immune cell marker CD14 (Ziegler-Heitbrock and Ulevitch, 1993) and CD44 (Schumann et al., 2015) (Fig. 6a). Cluster 14 and 15 have been assigned to RBC2 an RBC1 due to its expression of hemoglobin markers HBB (Talamo et al., 2003) and HBA1 (Pandey and Rizvi, 2011) (Fig. 6a). In addition to classical markers that were used to assign cell identities, Figure $6 a$ also includes novel markers we found for NC, AFC and NPC populations. A heatmap of the expression levels of top enriched genes for all sub-populations shows the high specificity of our enriched genes and the high cellular heterogeneity in IVD structures (Fig. S4). Collectively, the single-cell atlas of human IVD heterogeneously comprised of NC, NPC, AFC, IC, non-MC, and RBC populations, which can be more precisely classified into 15 different subtypes: NC1-2, NC/NPC, NPC1-4, iAFC, oAFC1-3, non-MC, IC and RBC1-2 (Fig. 6a).

The fully resolved single-cell atlases were plotted in three-dimensional (3D) UMPAs (Fig. 6b) for a more accurate and intuitive visualization. In the 3D atlas, NC1, NC2, IC, RBC, and non-MC visually appear to be away from the main continents. The NPC sub-populations (NPC1-4) tended to cluster together with vague boundaries whereas AFC sub-populations (iAFC, oAFC1-3) are distant from each other with clear boundaries.

One of the key pathways involved in the IVD is the Wnt/ $\beta$-catenin pathway (Kondo et al., 2011). We found that the Wnt/ $\mathrm{B}$-catenin pathway to be activated in neonatal NC but downregulated in neonatal and adult NPCs (Fig. 6c). We further expanded the key genes in the Wnt/ 3 -catenin pathway and showed the expression of SOX4 regulator (also the NC marker) was upregulated in neonatal NCs compared to neonatal and adult NPCs, whereas another regulator gene, SOX9, was downregulated in neonatal NCs compared to neonatal NPCs (Fig. 6d).

The Pseudo-time Trajectory Predicted Five Developmental States from Neonatal Cells into Adult Nucleus Pulposus Cells with Some Notochordal Cells Preserved Throughout Adulthood

The trajectory, color-coded by age, shows that neonatal cells occupy two branches and share one branch with the adult cells (Fig. 7a). Adult cells occupy the other two branches (Fig. 7a). ECM gene expression has changed over pseudo-time. COL1A1 expression decreased over pseudotime, while COL2A1 decreased in the early stage, but increased in the later stage. ACAN 
expression followed a swinging increase-decrease-increase pattern (Fig. 7b). SOX4, a positive regulator of the Wnt/ß-catenin canonical pathway (Sinner et al., 2007), decreased over pseudotime, but SOX9, a negative regulator (Sinha et al., 2021), increased (Fig. 7c), which is consistent with Fig. 6d.

NC populations are mainly positioned in the proximity to NPCs. NCs were present along the trajectory from the very early neonatal stage towards the later stage and eventually aggregate around NC/NPC in the adult branch (Fig. 7d). The two adult IVD-prevalent branches show very distinct cell compositions, one is mainly composed of NCs and NPCs, the other one is mainly composed of AFCs (Fig. 7d). The expression of novel and classical NC markers in the NC populations changed over pseudo-time, as shown in Fig. 7e. The expression of NPC markers in NPC populations also changed over pseudo-time (Fig. 7e). Specifically, COL2A1 decreased most dramatically over pseudo-time, but bounced back in the end, SOX9 and ACAN maintained relatively stable levels in NPC populations.

We found 7 cell states along the trajectory (Fig. 7f). The annotation of each state depends on the trajectories of age (Fig. 7a) and cell types (Fig. 7d). State 1 has been annotated as neonatal cells due to its mixed cell composition originated in neonatal IVD. State 2 has been annotated as neonatal NPC, as it is still in neonatal stage, but mostly consists of NPCs. State 3 has been annotated as NC to NPC, because it is comprised of both neonatal and adult cells and consists of both NCs and NPCs. State 4 and State 5 are two intermediate stages that contain NPCs, NCs, and AFCs. State 6 has been annotated as adult AFC, because it is dominated by adult cells, mainly of iAFCs and oAFCs. State 7 has been annotated as adult NPC, because it is dominated by adult NPCs with a small quantity of NCs and AFCs. The direction of trajectory is determined by the direction of pseudo-time. The change of expression levels of key molecules during the developmental process are also shown over pseudo-time in Fig. 7g, including SOX4, COL2A1, ACAN, COL1A1 and SOX4. Collectively, state $1 \mathrm{might}$ indicate an early developmental stage whereas state 2 and 3 may indicate the process of NCs developing into NPCs which may occur during the neonatal stage. State 6 might indicate that AFCs are present in both ages during the development, but they may develop from neonatal AFCs into adult AFCs. State 7 may indicate the development of large quantity of NPCs in adult that are likely originating from neonatal NCs. It also indicates the continuing presence of NCs in adult IVDs. 


\section{DISCUSSION}

This study provides the first direct comparison of cell atlases at single-cell level between neonatal and adult human IVDs. With the help of scRNA-seq technology, we discovered FABP4, AKR1C1, and $A P O E$ as novel markers for human NCs. Our data also supported the previous assumption of the presence of NCs in the adult. It also models the developmental process from neonatal IVD, composed of various cell types, into adult IVD, composed mainly of NPCs and AFCs. Lastly, AFCs in neonatal IVD were found to be highly heterogenous with a strong gradient profile that most probably correlated with spatial distribution.

This study identifies FABP4, AKR1C1, and APOE as highly specific markers for NC populations in the human neonatal and adult IVD (Fig. 3a, b). Although our network analysis demonstrated both $A K R 1 C 1$ and FABP4 to be associated with tumorgenicity and inflammation (Fig. 3c-e), these genes have also been associated with cell metabolism and may be therefore linked to increased cell activity and biosynthesis in early development. For example, AKR1C1 is an enzyme that catalyzes NAPDH-dependent reductions (Zeng et al., 2017) and is responsible for hormone secretion (Marín et al., 2009). FABP4 is expressed in embryonic cartilage and bone tissues (Urs et al., 2006). In addition, $A K R 1 C 1$ and FABP4 also mark the inflammatory response in early-stage development, as shown by VEGF-regulated FABP4 in neonatal NC1 sub-population that also activated CXCL8 (Fig. 3c). Similarly, AKR1C1 and APOE are involved in another IL1-acitivated, inflammation network (Fig. S1C). In previous research, the IL1 cascade was already considered an inflammatory and catabolic marker for adult and degenerative human IVD (Johnson et al., 2015). To our knowledge, its occurrence in neonatal IVD has not been previously reported. Noteworthy, FABP4 appears to be a robust NC1 marker in both neonatal and adult IVD. Although AKR1C1 can be used as NC2 marker, it is less specific in adult NC2 (Fig. 3a). This may indicate a reduced NC cell metabolism in the adult IVD compared to neonatal.

Our results show that $A P O E$ is highly specific to NCs in neonatal IVD (Fig. 2c), in contrast to a previous study in mouse IVD that demonstrated APOE to be expressed in both AFCs and NPCs (Zhang et al., 2013). Differences in findings may be due to the different species investigated. 
Isolating and identifying rare NC populations in adult IVD is a challenging task due to their small quantity. Our single-cell atlas detected rare NC populations in adult IVD expressing MAP1B marker, unveiled two distinct NC sub-populations, NC1 and NC2 in neonatal IVD, and confirmed their presence in adult IVD (Fig. 2a). To our knowledge, only one recent single-cell study by Gan et al. reported a NC population in adult IVD. However, they used TBXT to identify NCs, whereas we used MAP1B (Gan et al., 2021). Other studies, including proteomics (Tam et al., 2020b) and scRNA-seq (Fernandes et al., 2020) of human adult IVD, reported the presence of NPCs and AFCs, but did not report NC populations. The differences in findings might be a result of different samples analyzed or analysis techniques (Fernandes et al., 2020; Tam et al., 2020b). Partly in line with our study, Richardson et al. demonstrated the presence of a sub-population with NC-like phenotype but NPC-like morphology in adult IVD, using QPCR and immunohistochemical analysis (Richardson et al., 2017). In contrast to our findings (Fig. 2a), they detected an expression of $C D 24$ and TBXT. In a later study, Rodrigues-Pinto et al. from the same group reported the preselected CD24+ cells contained NCs expressing MAP1B (Rodrigues-Pinto et al., 2018), in fetal IVD. To our knowledge, our study is the only report demonstrating two distinct $M A P 1 B+N C$ subpopulations in adult IVD. Differences in results between Richardson et al., Rodrigues-Pinto et al. and our study might be due to several reasons: The work by Richardson et al. focused on the CD24+ sub-population as NC-like phenotype and observed their NP-like morphology (Richardson et al., 2017), while our study is focused MAP1B+ NC cells, since interestingly CD24+ cells were not identified by our analysis as a distinct cluster. Therefore, their CD24+ sub-populations may be a different, more heterogenous population across our clusters. Rodrigues-Pinto et al. sorted and specifically analyzed CD24+ cells (Rodrigues-Pinto et al., 2018), while our study did not include any pre-selection process and instead unbiasedly analyzed all cells. Lastly, our scRNAseq technique identified two NC populations, which we divided into NC1 and NC2 (Fig. 2a), which were not detected in previous studies (Fernandes et al., 2020; Gan et al., 2021).

Historically, NPCs are considered to be derived from NC lineage (Risbud et al., 2010). We confirmed the close lineage relations between NCs and NPCs and further unveiled the transition process. Firstly, our developmental trajectories predicted that NCs started to develop into NPCs even in the neonatal stage, and its continuing development resulted in a large quantity of NPCs 
in adult IVD (Fig. 7f). To the best of our knowledge, no previous study predicted the developmental trajectories crossing ages (early vs. late development) and cell types (NC versus NPC). One recent study predicted the differentiation of NPCs alone based on pseudo-time trajectories without specifying the actual ages (Gan et al., 2021), however, it did not include neonatal IVD that is rich in NCs. Secondly, we found significant distinctions between neonatal NC and adult NPC populations at molecular, cellular, and tissue levels. We showed that neonatal NCs have some tumor-like properties and are involved in tissue growth (Fig. 4d-f), as suggested by their expression of relevant genes (IL1B, VEGF, HGT, Fig. S1d) and enriched functions (Fig. 4d). In contrast, adult NPCs appear to be more mature, with closer association to fully differentiated cells and developed cytoskeletal tissues (Fig. 4d-f), and they expressed BMP2 and SOX9 (Fig. S1d). Our findings are in line with a previous IVD single-cell transcriptomic analysis showing BMP2 and SOX9 expression in some adult sub-populations (Gan et al., 2021). Changes were also observed in cell morphology. The adult NPCs have large cytoplasm, as opposed to the small neonatal NPCs (Fig. 1a, b). This is in line with the enriched function of development of cytoplasm for adult NPCs (Fig. 4d). The large cytoplasm of NPCs (Fig. 1b) contradicts a previous report stating that NCs are larger than NPCs (Rodrigues-Pinto et al., 2018). We found the matrix also changed from a fibrous, less-developed appearance to a more mature cartilage-like, (Fig. 1a, b), as well as the ECM gene expression transformed from a collagens-rich (COL1A1 and COL2A1) to aggrecanrich $(A C A N)$ in the later stage (Fig. 7b). It is known that COL2A1 ensures the removal of notochord during the development (Aszódi et al., 1998). Thus, the decrease of COL2A1 (Fig. 7b, g) may mark the successful removal of notochord and completion of NC to NPC transition. These distinctions at molecular, cellular, and tissue levels highlight the changes occur in neonatal NC during their development into adult NPC. Though most NCs differentiated into adult NPCs, some NCs kept their identity throughout the entire trajectory (Fig. 7d). The NC quantity is significantly decreased in adult (NCs constituted $7.5 \%$ of total cell counts in neonatal IVD and $17.5 \%$ in adult IVD, data not shown). Previous studies were not conclusive on their presence (Fernandes et al., 2020; McCann and Séguin, 2016; Risbud and Shapiro, 2011; Séguin et al., 2018; Wang et al., 2008). It should be noted that NC1 almost disappeared in adult IVD but NC2 percentage significantly elevated (Fig. 1h). This indicates that NCs became more involved in ECM interactions and tissue developments but maintained robust pluripotency during the development. (Fig. S1a and S1b). Our study shows that the NCs kept expressing NC markers, MAP1B and AKR1C1, in 
later pseudo-time and even elevated in the very late stage (Fig. 7e). Therefore, the NCs may have preserved their capability to differentiate into NPCs during their continuing presence of NCs throughout the adulthood.

The high heterogeneity of AFCs found in neonatal IVD is in line with recent studies that also described spatially defined AFC populations divided into inner and outer regions (Shi et al., 2015; Tam et al., 2020a). Our study presented a very detailed classification of AFCs into one subpopulation to inner AF regions (iAFC), and three distinct sub-populations in the outer AF regions (oAFC1-3), which has not been reported in previous relevant studies (Shi et al., 2015; Tam et al., 2020a).

A striking trend of decreasing expression levels of collagen-associated genes (Fig. $5 d$ ) appeared when the order of the five AFC sub-populations from neonatal IVD was re-arranged from the inner region to the outer region to iAFC, oAFC1, oAFC2, oAFC3 (Fig. 5d). Interestingly, another proteomics study by Tam et al. (Tam et al., 2020b) showed ECM proteins in 16- to 17-year-olds increased from the inner NP to the outer AF region of the IVD, which seems to be contradictory to our findings of the decreasing expression of collagen-producing genes (Fig. $5 \mathrm{~d}$ ). The protein content and RNA level may not necessarily be aligned. In line with Tam et al., we found GP6 signaling pathway relevant to cell-ECM interaction show gradient of expression from inner AF to outer AF (Fig. 5e). The oAFC and iAFC have distinct gene expression profiles possibly due to their different spatially defined roles. AFCs are in a position that connects endplate and inner NP core, as suggested in Fig. 5f. The promoted Unfolded Protein Response (UPR) we detected in the outer AFC region (Fig. 5e) may be a sign of external stressors (Hetz et al., 2020; Wang et al., 2010). The UPR also orchestrates the enforcement of adaptive mechanisms to maintain an optimal rate of protein production and rapidly reacts to diverse stimuli, including extracellular responses to hormones, growth factors and small ligands that bind cell-surface receptors (Hetz et al., 2020). UPR is also a major contributor of the CALR marker for oAFC sub-populations (Fig. S2). CALR was previously found to be expressed in the oAF as well (Tam et al., 2020b) and it is a key gene in UPR pathway (Fig. S2). In addition to UPR signaling, the platelet-derived growth factor (PDGF) signaling also follows the increasing trend from inner to outer AF regions (Fig. 5f). PDGF expression has been shown to promote the proliferation of AFCs and NPCs (Pratsinis and Kletsas, 2007), suggesting a potential increased cell proliferation in the outer region. In short, the 
UPR and PDGF signaling may indicate an increased cell proliferation in response to external stimuli and stress applied to the outer AFCs. This theory was additionally supported by our detection of the expressed heat shock protein, HSPA6, in the outmost oAFC3 sub-population. Heat shock proteins (HSPs, Fig. 5a) were previously found to be strongly expressed in the outmost area of IVD and endplate cartilage in response to mechanical stress (Takao and Iwaki, 2002; Tam et al., 2020a). Collectively, the gradient trend of AFCs in neonatal IVD might be associated with certain biological function, for instance the elevated stress-induced cell proliferation and remodeling process among oAFCs. However, this needs to be confirmed by spatial single-cell analysis in the future.

The present work is not without limitations: our scRNA-seq lacks spatial resolution. The recently emerging single-cell spatial sequencing technology may be required in the next study.

In conclusion, we identified FABP4, AKR1C1 and APOE as novel markers for human NC populations. We demonstrated the first direct comparison of human neonatal and adult IVD at a single-cell level, finding two distinct NC sub-populations in both neonatal and adult IVD. A pseudotime trajectory analysis predicted the developmental process from neonatal IVD, composed of various cell types, into adult IVD, composed of mainly NPCs and AFCs. We also found that NCs preserve their cell identity, and some of their markers and function into adulthood. Lastly, we demonstrated a high heterogeneity of AFC populations in neonatal IVD and showed their gradual transitions of cell types, gene expression, function, signaling pathways, as well as their potential relevance in IVD development, which appears to be linked to a spatial gradient. Better understanding of these processes may help to identify the underlying factors contributing to ageassociated diseases of the IVD and to develop tailored therapeutics that may delay or reverse these processes. 


\section{STAR METHODS}

\section{Key Resource Table}

\begin{tabular}{|c|c|c|}
\hline REAGENT or RESOURCE & SOURCE & IDENTIFIER \\
\hline \multicolumn{3}{|l|}{ Antibodies } \\
\hline Rabbit Polyclonal Anti-HSPA6 & Abcam & ab212044 \\
\hline Mouse Monoclonal Anti-CD44 (Clone Bu52) & Biorad & MCA2504T \\
\hline Mouse Monoclonal Anti-SOX17 & Invitrogen & MA5-24885 \\
\hline Mouse Monoclonal Aldo-keto Reductase 1C1/AKR1C & R\&D Systems & MAB6529-SP \\
\hline Goat Polyclonal Anti-FABP4/A-FABP & R\&D Systems & AF3150-SP \\
\hline Rabbit Monoclonal Anti-Apolipoprotein E [EP1374Y] & Abcam & ab52607 \\
\hline Polyclonal Rabbit Anti-MAP1B & Novus Biologicals & NBP3-04801-20ul \\
\hline \multicolumn{3}{|l|}{ Bacterial and virus strains } \\
\hline & & \\
\hline & & \\
\hline & & \\
\hline \multicolumn{3}{|l|}{ Biological samples } \\
\hline Human neonatal IVD & NDRI & ND18842 \\
\hline Human adult IVD & $\begin{array}{l}\text { Cedars-Sinai Medical } \\
\text { Center }\end{array}$ & Patient Cadaver \\
\hline \multicolumn{3}{|l|}{ Chemicals, peptides, and recombinant proteins } \\
\hline Chromium Single-cell 3' Reagent Kits & 10x Genomics & \\
\hline DAPI (4',6-Diamidino-2-Phenylindole, Dihydrochloride) & Invitrogen & D1306 \\
\hline \multicolumn{3}{|l|}{ Critical commercial assays } \\
\hline & & \\
\hline & & \\
\hline & & \\
\hline \multicolumn{3}{|l|}{ Deposited data } \\
\hline & & \\
\hline
\end{tabular}




\begin{tabular}{|c|c|c|}
\hline \multicolumn{3}{|l|}{ Experimental models: Cell lines } \\
\hline & & \\
\hline & & \\
\hline \multicolumn{3}{|c|}{ Experimental models: Organisms/strains } \\
\hline & & \\
\hline & & \\
\hline & & \\
\hline & & \\
\hline \multicolumn{3}{|l|}{ Oligonucleotides } \\
\hline & & \\
\hline & & \\
\hline & & \\
\hline \multicolumn{3}{|l|}{ Recombinant DNA } \\
\hline & & \\
\hline & & \\
\hline & & \\
\hline \multicolumn{3}{|l|}{ Software and algorithms } \\
\hline ImageJ (v2.1.0/1.53c) & $\begin{array}{l}\text { (Schneider et al., } \\
\text { 2012) }\end{array}$ & \\
\hline Fiji & $\begin{array}{l}\text { (Schindelin et al., } \\
\text { 2012) }\end{array}$ & \\
\hline $\mathrm{R}(\mathrm{v} 4.0 .3)$ & $\begin{array}{l}\text { R Development Core } \\
\text { Team }\end{array}$ & $\begin{array}{l}\text { https://www.R- } \\
\text { project.org }\end{array}$ \\
\hline RStudio (v1.4.1103) & $\begin{array}{l}\text { RStudio Team (2020). } \\
\text { RStudio: Integrated } \\
\text { Development for R. } \\
\text { RStudio, PBC }\end{array}$ & $\begin{array}{l}\text { https://www.rstudio.c } \\
\text { om/products/rstudio/ }\end{array}$ \\
\hline Cell Ranger (v3.0.0) & 10x Genomics & \\
\hline Loupe Cell Browser (v3.0.0) & 10x Genomics & \\
\hline
\end{tabular}




\begin{tabular}{|l|l|l|}
\hline Seurat (v4.0.1) & $\begin{array}{l}\text { (Butler et al., 2018; } \\
\text { Hao et al., 2021; Satija } \\
\text { et al., 2015; Stuart et } \\
\text { al., 2019) }\end{array}$ & $\begin{array}{l}\text { https://satijalab.org/s } \\
\text { eurat/ }\end{array}$ \\
\hline Monocle (v2.18.0) & $\begin{array}{l}\text { (Qiu et al., 2017a; Qiu } \\
\text { et al., 2017b; Trapnell } \\
\text { et al., 2014) }\end{array}$ & $\begin{array}{l}\text { http://cole-trapnell- } \\
\text { lab.github.io/monocl } \\
\text { e-release/ }\end{array}$ \\
\hline Other & & \\
\hline & & \\
\hline & & \\
\hline & & \\
\hline
\end{tabular}

\section{RESOURCE AVAILABILITY}

\section{Lead contact}

Further information and requests for resources and reagents should be directed to and will be fulfilled by the lead contact, Dmitriy Sheyn (dmitriy.sheyn@csmc.edu)

\section{Materials availability}

This study did not generate new unique reagents.

\section{Data and code availability}

The raw sequencing data and original data are accessible in repositories. 


\section{EXPERIMENTAL MODEL AND SUBJECT DETAILS}

The Cedars-Sinai Medical Center Institutional Review Board approved this study (IRB number Pro00052234). Human neonatal and adult IVDs have both been investigated with scRNA-seq to reveal their cell atlas of respective age and to determine the difference between them. The neonatal samples were collected from 4 different spine levels (L1-L5) from a 6-hour postnatal male provided by the National Disease Research Interchange (NDRI, ND18842), 3 levels were used for the scRNAseq analysis and one for histology. The adult samples were collected from lumbar discs of 2 cadaveric spines and 1 clinical sample of removed and otherwise discarded disc. IVD tissues were harvested from three neonatal spinal disc levels and adult cadavers or clinical samples of patients with no back pain history (over 65 years old) were collected and analyzed via scRNA-seq ( $n=3$ for neonatal IVDs, $n=3$ for adult IVDs). Additional lumbar levels were processed for histological analysis.

\section{METHOD DETAILS}

\section{Single-cell Isolation and Sequencing}

The IVDs were dissected and washed with Phosphate Buffered Saline (PBS). The tissue was manually minced to $\sim 1 \mathrm{~mm}$ pieces in a sterile environment and enzymatically digested. Tissue was digested for 1 hour at $37^{\circ} \mathrm{C}$ in $2 \mathrm{mg} / \mathrm{mL}$ of Pronase Protease (Millipore, Temecula, CA) in growth media containing $1 \mathrm{mM} \mathrm{L-glutamine,} \mathrm{1 \%} \mathrm{antibiotic-antimycotic} \mathrm{solution} \mathrm{(Gibco,} \mathrm{Carlsbad,}$ CA) and 10\% fetal bovine serum (FBS, Gemini Bioproducts, West Sacramento, CA) in Dulbecco's modified eagle media-F12 media (DMEM/F12, GIBCO, Carlsbad, CA). This was followed by $\sim 18$ hours digestion at $37^{\circ} \mathrm{C}$ in $0.25 \mathrm{mg} / \mathrm{mL}$ of Collagenase Type $1 \mathrm{~S}$ (Sigma Aldrich) in growth media. The resulting sample was pushed through a $70 \mu \mathrm{m}$ cell strainer and cells were isolated via centrifugation at $1200 \mathrm{rpm}$ for ten minutes, The pellet was resuspended in phosphate buffered saline at a concentration of $1,500 \mathrm{cells} / \mu \mathrm{L}$ and $100 \mathrm{uL}$ of sample was processed for single-cell RNA sequencing using the Chromium Single-cell 3' Reagent Kits from 10X Genomics. 
Chromium Single-cell 3' v2 libraries with $\sim 3,000$ cells were prepared on a Chromium Controller with chips and reagents from Single-cell Gene Expression v2 kits following the manufacturer's protocols (10x Genomics). The libraries were then sequenced using paired-end sequencing (28bp Read 1 libraries, and $91 \mathrm{bp}$ Read 2) with a single sample index (8bp) on an Illumina NovaSeq. Samples were sequenced to a depth of $>50,000$ raw reads per cell, with raw sequencing data analyzed and visualized with pre-release versions of Cell Ranger 3.0.0 and Loupe Cell

Browser 3.0.0. Raw sequencing data was demultiplexed and converted to fastq format by using bcl2fastq v2.20 (Illumina, San Diego, CA). Fragment analysis of indexed libraries was performed on the Agilent 4200 TapeStation (Agilent Technologies, Santa Clara, CA).

Integration, Pre-processing, and Dimensional Reduction of the Single-cell Dataset of Neonatal and Adult IVD

Seurat package (v4.0.1) in R (v4.0.3) has been used to process the data of four samples. We firstly used CreateSeuratObject function to transform the loaded data into a Seurat object for each sample. Next, the four Seurat objects were combined into one matrix. NormalizeData and FindVariableFeatures were used. Afterwards, FindIntegrationAnchors and IntegrateData were used to integrate and anchor the 6 samples' data together. RunPCA function was used to for principal component analysis with $\mathrm{PC}=30$. DimPlot function was then used with "UMAP" reduction to obtain the 2D Uniform Manifold Approximation and Projection (UMAP) plot. FindNeighbors and FindClusters functions further classified all cells in 6 samples into 15 clusters at resolution of 0.5 (unsupervised clustering). The UMAP was then split by their sample type (neonatal or adult IVD) for comparison.

\section{Detection of Enriched Genes and Novel Markers for Notochordal Cells}

The expression levels of NC relevant genes in neonatal or adult IVD were separately visualized using violin plot in log scale with split view. In the volcano plots, we set the cutoff threshold of FC to be $>2$ and the cutoff threshold of adjusted $p$ value to be $<1 \times 10^{-24}$. Any genes falling out of this 
range were not considered as enriched genes or markers. Genes falling into this range were labeled with red color in the volcano plot. Next, top enriched genes from the red colored area were manually selected as novel in silico markers for specific cluster from neonatal IVD or adult IVD. Their expression levels were presented quantitatively in violin plots.

Visualization of Single-Cell Atlases with 3D UMAP Plots

The cell coordination of the top three dimensions (PC1, PC2, and PC3) were extracted from the Seurat object integrating neonatal IVD and adult IVD. FetchData function was used to create the data frame for the 3D UMAP plot. The plot_ly function in Plotly package was used to obtain the final 3D UMAP plot. The color for each type was plot as the same as in 2D UMAP.

\section{Gene Ontology Term Enrichment and Pathway Analysis}

Gene ontology (GO) term enrichment and pathway analysis was performed using Qiagen Ingenuity Pathway Analysis (IPA, version 65367011). A list of enriched genes for a specific cell sub-population (as compared against all other cells) was firstly generated in $\mathrm{R}$ studio using FindMarkers function. The specific cell sub-populations include NC1-2, iAFC, oAFC1-3 respectively. In other attempts, we did multiple further analyses: we created enriched gene list for NC by combining NC1 and NC2 cell sub-populations followed by FindMarkers function against all other cells; we created enriched gene list for NPC by combining NPC1-4 cell sub-populations followed by FindMarkers function against all other cells. All the above enriched gene lists were generated for both neonatal IVD and adult IVD. The adjusted $p$ value and fold change for genes with $p<0.0001$ were loaded into the IPA software. We included the database for human tissue and primary cells but excluded those for cell lines, as our samples were harvested from human samples only. The analyses covered canonical pathways, upstream analysis, diseases and biological functions, and regulatory networks. The heatmaps for enriched biological functions, enriched pathways, and key genes in the pathways were replotted using GraphPad Prism (v9.2.0). We demonstrated the most relevant results in figures. 
The comparison of gene overlap in NCs and NPCs in both neonatal and adult IVD was performed at Metascape.org using Circos plot. The enriched genes with $p<0.0001$ and $\mathrm{FC}>1$ were selected for Circos plot analysis.

Histology and Immunofluorescence

IVD samples from the sample were fixed in $10 \%$ phosphate buffered formalin for three days, decalcified by incubation in 0.5M EDTA ( $\mathrm{pH} 7.4$, Sigma Aldrich) for three weeks, and embedded in paraffin. Five-micron-thick sections were cut from the paraffin blocks. Hematoxylin and eosin (H\&E) staining was performed to evaluate the morphological features. Images were captured using a Carl Zeiss Axio Imager Z1 fluorescent microscope (Carl Zeiss, Germany) equipped with ApoTome and AxioCam HRc cameras. Images were analyzed using QuPath software (v0.2.3).

For immunofluorescence, sections were deparaffinized, rehydrated in DPBS, treated with antigen retrieval solution at room temperature for 20 minutes (Dako \# S3020, Agilent Technologies). Slides were washed with PBS, blocked with 3\% normal donkey serum (Jackson ImmunoResearch Laboratories Inc., West Grove, PA) in 0.3\% Triton-X (Sigma Aldrich, St. Louis, MO) and again washed with PBS. Slides were stained with primary antibodies (anti-human HSPA6, CD44, SOX17, AKR1C1, FABP4, APOE). The primary antibodies were applied to the slides, after which the slides were incubated at $4^{\circ} \mathrm{C}$ overnight and washed using PBS; the slides were then incubated with secondary antibodies for $1 \mathrm{hr}$ at room temperature. Finally, the slides were stained with 4',6diamidino-2-phenylindole dihydrochloride (DAPI, $0.28 \mu \mathrm{g} / \mathrm{ml}$ ) for $5 \mathrm{~min}$ in the dark. Subsequently, sections were washed three times in dPBS and mounted with Prolong Gold with DAPI (Life Technologies).

Pseudo-Time Analysis of Developmental Trajectories 
The pseudo-time developmental trajectory analysis was performed using Monocle package (v2.18.0). The original integrated Seurat object was subset into a new Seurat object that contains the following cell populations: NC (as the combination of NC1-2), NPC (as the combination of NPC1-4), iAFC, and oAFC (as the combination of oAFC1-3). Low-quality cells were filtered by the following standards: 1) Cells whose total mRNAs $>10^{6}$ were filtered; 2) Cells whose total mRNAs were out of a range of mean $\pm 2 \times$ standard deviations were filtered. The gene marker was also filtered out when less than 10 cells expressed this maker. The data matrix was then logtransformed and standardized to the same scale. We chose up to 500 top globally enriched genes for each sub-populations to determine cell progress. The dimensions of the dataset were reduced using DDRTree method. The trajectories were plotted using plot_cell_trajectory function. The cells in trajectories were colored with either their ages, cell types, or pseudo-time states. 


\section{ACKNOWLEDGMENTS}

This study was supported by NIH/NIAMS, Grant/Award Number: K01AR071512. The authors wish to acknowledge the Cedars-Sinai Genomics Core for next generation services and the Cedars-Sinai Biobank for human adult cadaver sample. The authors thank National Disease Research Interchange (NDRI) for human neonatal sample and Mrs. Julia Sheyn for the fluorescent images acquisition.

\section{AUTHORS CONTRIBUTIONS}

Conceptualization, W.J., D.S., J.G., and R.H.; Methodology, W.J., R.H., D.S., P.M., and A.W.; Software, W.J., R.H., and A.W.; Validation, K.S and G.K.; Formal Analysis, W.J., R.H., and D.S.; Investigation, W.J., D.S., and R.H.; Resources, R.H., D.S. and W.J.; Data Curation, W.J. and R.H.; Writing - Original Draft, W.J.; Writing Review \& Editing, J.G., D.S., W.J., R.H., and G.K.; Visualization, W.J., D.S., and R.H.; Supervision, D.S., and R.H.; Funding Acquisition, D.S.

\section{DECLARATION OF INTERESTS}

The authors declare no competing interests. 
FIGURE LEGENDS

Figure 1. Sorting of Cells from Human Neonatal and Adult IVD of Distinct Tissue Morphologies into 15 Unsupervised Clusters Based on Their Expression of IVD Markers. H\&E staining of a) neonatal IVD and b) adult IVD. The black dashed line shows the boundary separating two distinct regions in a). Black arrow points to a blood vessel. The white arrows point to NPCs. c-d) UMAP shows 15 unsupervised cell clusters that were identified in both neonatal (c) and adult (d) samples. e-f) Dot plots showing the expression level of classical IVD markers in each cluster of neonatal (e) and adult (f) IVD. Dot size indicates percent of cells expressing the genes and dot color intensity the average expression level (normalized to -2 to 2). g) Combined UMAP for all cells from neonatal and adult IVDs with clusters assigned to sub-populations based on their marker expressions in (c-d). h) Cell distribution between two ages (neonatal and adult) for each sub-population. Numbers were normalized to total cell counts for each age.

Figure 2. Identification of NC Sub-Populations and Their Pathway Network Analysis. a) The two NC sub-populations in UMAP and their expression of classical markers in both neonatal and adult IVD; two MAP1B+/SOX4+ NC sub-populations in the UMAP are colored green, and all other cells are colored grey; violin plots show NC expression levels of classical markers of NCs and immune cells for human neonatal and adult IVD. Other cells refer to the expression level for all cells in all clusters other than NC1 and NC2. b) The immunostaining of classical NC and immune markers in neonatal and adult IVD are shown. Scale bar $=100 \mu \mathrm{m}$; original magnification $=20 \mathrm{x}$. c) Volcano plots showing the enriched genes for the entire populations of NC, sub-populations of NC1 or NC2, in neonatal or adult IVD. The y-axis in the volcano plots shows the $-\log _{10} p$ where the $p$ value was about an enriched gene expression level in a specific sub-population in a specific age (neonatal or adult) against all other cells the same age. The $x$-axis shows the $\log _{2} F C$ (FC fold change) of the expression level. The cut-off threshold was set to FC>2 and $p<1 \times 10^{-24}$. The enriched genes meeting the cut-off threshold are colored with red. The genes not meeting the cut-off threshold are colored with grey. 
Figure 3. Discovery of Novel NC Markers, FABP4, AKR1C1, APOE, and Their Pathway/Network Analysis. a) Comparison of the expression level of novel top markers in human neonatal versus adult IVD. b) Immunostaining of novel NC markers in neonatal and adult IVD are shown. The scale bar $=100 \mu \mathrm{m}$ and original magnification $=20 x$. Qiagen IPA software was used to plot the c) VEGF-regulated network involving FABP4 marker in NC1 sub-population in neonatal IVD and P38-MAPK-regulated network involving FABP4 marker in NC1 subpopulation in adult IVD. d) TGFB1-regulated network involving AKR1C1 marker in NC2 subpopulation in neonatal IVD. e) Top biological functions activated by $A K R 1 C 1 / A K R 1 C 2$ and ranked by the activation score in NC1 sub-population in neonatal IVD.

Figure 4. Identification of 4 Similar NPC Sub-Populations and Comparison of Their NPC Gene Expression, Pathways, and Biofunctions with NC Populations in Neonatal and Adult IVD. a-b) Violin plots showing differences in the expression levels of NPC markers in four NPC sub-populations (NPC1-4) identified among the ACAN+/COL2A1+/SOX9+ NPC populations in UMAP (colored in blue) in both neonatal (a) and adult IVD (b). AFC markers were shown for comparison. Expression levels of each NPC subtype marker were projected via UMAP. c) Immunostaining of extracellular matrix (ECM) proteins in neonatal and adult IVD is shown. Scale bar $=100 \mu \mathrm{m}$; Original magnification $=20 \mathrm{x} . \mathrm{d}$ ) Heatmap shows biological functions enriched from all DEGs of NCs or NPCs in neonatal or adult IVD. Orange indicates activation, deactivation, and grey data that were not detected or did not pass the filtering. e) Circos plot showing the overlap between four different enriched gene lists: neonatal NCs, adult NCs, neonatal NPCs, and adult NPCs. Each purple line links the same gene expressed in both enriched gene list. Red and orange show the shared and unique genes. f) Descriptive summary terms on the biofunctions enriched from each list.

Figure 5. Heterogeneity of Annulus Fibrosus Cells in Human Neonatal and Adult IVDs. a-b) Expression levels of classical AFC markers (COL1A1, CALR, HSPA6), classical NPC markers (ACAN, COL2A1, SOX9) and classical NC markers (MAP1B) are shown for comparison, for both (a) neonatal IVD and (b) adult IVD. c) The AFC populations showed strong heterogeneity in 
neonatal IVD by having 4 distinct subtypes and their heterogenous markers. We demonstrated the expression distribution projected on UMAP and their quantitative expression levels. d) Among the 5 heterogenous sub-populations detected in (c), the neonatal AFCs exhibited a decreasing trend of expression levels of ECM-relevant, collagen-producing genes from inner core NPCs to the outer region AFCs following the order of iAFC, oAFC1-3. e) The biological function and key canonical pathway enrichment results followed the same gradient. f) Predicted scheme for spatial distribution of the 4 AFC populations, along with inner NPC core and notochord, in neonatal IVD, as compared with the classical IVD structure in adult human.

Figure. 6. Complete Single-cell Atlases of Human Neonatal and Adult IVD. a) Complete dot plots of marker expression of all sub-populations in the neonatal and adult IVD, including classical markers and novel markers found in the present study. The percent expressed is shown by the size of the dot, and the average expression level (normalized to -2 to 2 ) is shown by the color scale. b) Complete single-cell atlases visualized with 3D UMAP method, for both neonatal and adult IVD. The gene markers in the green rectangle are the novel markers found in the present study. c) Activation Z-score rated by Qiagen IPA software showing the canonical Wnt/ $\beta$-catenin pathway has been differentially regulated in NCs and NPCs in neonatal or adult IVD. d) Wnt/ $\beta$ catenin pathway key molecules' expression levels are shown in the heatmap. The orange indicates the activation, the blue indicates the deactivation, and the grey indicates the data either was not detected or did not pass the filtering.

Figure 7. Pseudo-time Trajectories Predicted IVD Cellular and Molecular Transition During Development. a) Cell trajectories predicted based on 500 top genes enriched in each subpopulation and color-coded based on different ages. b-c) The pseudo-time transition of b) ECM markers and c) Wnt/ $\beta$-catenin canonical pathway regulators. d) Cell trajectories color-coded by different cell types, including NC, NPC, iAFC, and OAFC, and e) the change of classical and novel NC markers in NCs over pseudo-time, and change of classical NPC markers in NPCs over pseudo-time. f) Cell trajectories color-coded by different cell states. Solid grey line shows the predicted developmental direction, and the dashed grey line shows the possible but inconclusively 
predicted developmental direction. g) Pseudo-time transition of molecules of interest are displayed and colored with cell states.

Figure S1. Additional Pathway and Network Analyses for NC Sub-Populations and Key Regulators in NC Populations Compared with NPC Populations in Neonatal and Adult IVD. Pathway and network summary for a) NC1 and b) NC2 sub-populations in neonatal IVD. c) The IL1-regulated network involving $A P O E$ and $A K R 1 C 1 / A K R 1 C 2$. d) Heatmap showing top regulators for NCs and NPCs in neonatal and adult IVD. Orange indicates activation, blue deactivation, and grey shows data that were either not detected or did not pass the filtering.

Figure S2. Gene Expression Projected on the Spatially Dependent Unfolded Protein Response Signaling Pathway for oAFC2 Sub-Populations in Neonatal IVD.

Figure S3. Comparison of the Spatially Dependent Trend of Expression Levels for Collagen-Relevant Genes in Neonatal and Adult IVD.

Figure S4. Heatmap Showing the Transcriptomes of Top Differentially Expressed Genes for All Sub-Populations Identified in Human IVD. Data include both neonatal and adult IVD. 


\section{REFERENCES}

Andersson, G.B. (1999). Epidemiological features of chronic low-back pain. Lancet 354, 581-585. 10.1016/S0140-6736(99)01312-4.

Aszódi, A., Chan, D., Hunziker, E., Bateman, J.F., and Fässler, R. (1998). Collagen II Is Essential for the Removal of the Notochord and the Formation of Intervertebral Discs. Journal of Cell Biology 143, 1399-1412. 10.1083/jcb.143.5.1399.

Bhattaram, P., Penzo-Méndez, A., Sock, E., Colmenares, C., Kaneko, K.J., Vassilev, A., DePamphilis, M.L., Wegner, M., and Lefebvre, V. (2010). Organogenesis relies on SoxC transcription factors for the survival of neural and mesenchymal progenitors. Nature Communications 1, 9. 10.1038/ncomms1008.

Bi, W., Deng, J.M., Zhang, Z., Behringer, R.R., and de Crombrugghe, B. (1999). Sox9 is required for cartilage formation. Nature Genetics 22, 85-89. 10.1038/8792.

Buckley, C.T., Hoyland, J.A., Fujii, K., Pandit, A., latridis, J.C., and Grad, S. (2018). Critical aspects and challenges for intervertebral disc repair and regeneration-Harnessing advances in tissue engineering. JOR SPINE 1, e1029. 10.1002/jsp2.1029.

Butler, A., Hoffman, P., Smibert, P., Papalexi, E., and Satija, R. (2018). Integrating single-cell transcriptomic data across different conditions, technologies, and species. Nature Biotechnology 36, 411-420. 10.1038/nbt.4096.

Cai, H., Zou, J., Wang, W., and Yang, A. (2021). BMP2 induces hMSC osteogenesis and matrix remodeling. Mol Med Rep 23, 125. 10.3892/mmr.2020.11764.

Chen, W.K., Yu, X.H., Yang, W., Wang, C., He, W.S., Yan, Y.G., Zhang, J., and Wang, W.J. (2017). IncRNAs: novel players in intervertebral disc degeneration and osteoarthritis. Cell Prolif 50. 10.1111/cpr.12313. 
D'Amour, K.A., Agulnick, A.D., Eliazer, S., Kelly, O.G., Kroon, E., and Baetge, E.E. (2005). Efficient differentiation of human embryonic stem cells to definitive endoderm. Nature Biotechnology 23, 1534-1541. 10.1038/nbt1163.

de Schepper, E.I., Damen, J., van Meurs, J.B., Ginai, A.Z., Popham, M., Hofman, A., Koes, B.W., and Bierma-Zeinstra, S.M. (2010). The association between lumbar disc degeneration and low back pain: the influence of age, gender, and individual radiographic features. Spine (Phila $\mathrm{Pa}$ 1976) 35, 531-536. 10.1097/BRS.0b013e3181aa5b33.

Fernandes, L.M., Khan, N.M., Trochez, C.M., Duan, M., Diaz-Hernandez, M.E., Presciutti, S.M., Gibson, G., and Drissi, H. (2020). Single-cell RNA-seq identifies unique transcriptional landscapes of human nucleus pulposus and annulus fibrosus cells. Sci Rep 10, 15263. 10.1038/s41598-020-72261-7.

Frank, J.W., Kerr, M.S., Brooker, A.S., DeMaio, S.E., Maetzel, A., Shannon, H.S., Sullivan, T.J., Norman, R.W., and Wells, R.P. (1996). Disability resulting from occupational low back pain. Part I: What do we know about primary prevention? A review of the scientific evidence on prevention before disability begins. Spine (Phila Pa 1976) 21, 2908-2917. 10.1097/00007632-19961215000024.

Frapin, L., Clouet, J., Delplace, V., Fusellier, M., Guicheux, J., and Le Visage, C. (2019). Lessons learned from intervertebral disc pathophysiology to guide rational design of sequential delivery systems for therapeutic biological factors. Advanced Drug Delivery Reviews 149-150, 49-71. https://doi.org/10.1016/j.addr.2019.08.007.

Gan, Y., He, J., Zhu, J., Xu, Z., Wang, Z., Yan, J., Hu, O., Bai, Z., Chen, L., Xie, Y., et al. (2021). Spatially defined single-cell transcriptional profiling characterizes diverse chondrocyte subtypes and nucleus pulposus progenitors in human intervertebral discs. Bone Research 9, 37. 10.1038/s41413-021-00163-z.

Hao, Y., Hao, S., Andersen-Nissen, E., Mauck, W.M., III, Zheng, S., Butler, A., Lee, M.J., Wilk, A.J., Darby, C., Zager, M., et al. (2021). Integrated analysis of multimodal single-cell data. Cell 184, 3573-3587.e3529. 10.1016/j.cell.2021.04.048.

Hetz, C., Zhang, K., and Kaufman, R.J. (2020). Mechanisms, regulation and functions of the unfolded protein response. Nature Reviews Molecular Cell Biology 21, 421-438. 10.1038/s41580020-0250-z. 
Ho, R., Workman, M.J., Mathkar, P., Wu, K., Kim, K.J., O’Rourke, J.G., Kellogg, M., Montel, V., Banuelos, M.G., Arogundade, O.A., et al. (2021). Cross-Comparison of Human iPSC Motor Neuron Models of Familial and Sporadic ALS Reveals Early and Convergent Transcriptomic Disease Signatures. Cell Systems 12, 159-175.e159. 10.1016/j.cels.2020.10.010.

Huang, Y., and Mahley, R.W. (2014). Apolipoprotein E: structure and function in lipid metabolism, neurobiology, and Alzheimer's diseases. Neurobiol Dis 72 Pt A, 3-12. 10.1016/j.nbd.2014.08.025.

Hunter, C., Matyas, J., and Duncan, N. (2003). The notochordal cell in the nucleus pulposus: a review in the context of tissue engineering. Tissue engineering 9, 667-677.

Johnson, Z.I., Schoepflin, Z.R., Choi, H., Shapiro, I.M., and Risbud, M.V. (2015). Disc in flames: Roles of TNF- $\alpha$ and IL-1 $\beta$ in intervertebral disc degeneration. European cells \& materials 30, 104117. 10.22203/ecm.v030a08.

Kelly, N.H., Huynh, N.P.T., and Guilak, F. (2020). Single cell RNA-sequencing reveals cellular heterogeneity and trajectories of lineage specification during murine embryonic limb development. Matrix Biology 89, 1-10. https://doi.org/10.1016/j.matbio.2019.12.004.

Knezevic, N.N., Mandalia, S., Raasch, J., Knezevic, I., and Candido, K.D. (2017). Treatment of chronic low back pain-new approaches on the horizon. Journal of pain research 10, 1111.

Kondo, N., Yuasa, T., Shimono, K., Tung, W., Okabe, T., Yasuhara, R., Pacifici, M., Zhang, Y., Iwamoto, M., and Enomoto-Iwamoto, M. (2011). Intervertebral disc development is regulated by Wnt/B-catenin signaling. Spine 36, E513-E518. 10.1097/BRS.0b013e3181f52cb5.

Lu, H., Cassis, L.A., Kooi, C.W.V., and Daugherty, A. (2016). Structure and functions of angiotensinogen. Hypertension Research 39, 492-500. 10.1038/hr.2016.17.

Lu, S.-Y., Li, M., and Lin, Y.-L. (2010). Mitf induction by RANKL is critical for osteoclastogenesis. Mol Biol Cell 21, 1763-1771. 10.1091/mbc.e09-07-0584.

Macfarlane, G.J., Thomas, E., Croft, P.R., Papageorgiou, A.C., Jayson, M.I.V., and Silman, A.J. (1999). Predictors of early improvement in low back pain amongst consulters to general practice: 
the influence of pre-morbid and episode-related factors. Pain 80, 113-119. https://doi.org/10.1016/S0304-3959(98)00209-7.

Marín, Y.E., Seiberg, M., and Lin, C.B. (2009). Aldo-keto reductase 1C subfamily genes in skin are UV-inducible: possible role in keratinocytes survival. Experimental Dermatology 18, 611-618. https://doi.org/10.1111/j.1600-0625.2008.00839.x.

Mathys, H., Davila-Velderrain, J., Peng, Z., Gao, F., Mohammadi, S., Young, J.Z., Menon, M., He, L., Abdurrob, F., Jiang, X., et al. (2019). Single-cell transcriptomic analysis of Alzheimer's disease. Nature 570, 332-337. 10.1038/s41586-019-1195-2.

McCann, M.R., and Séguin, C.A. (2016). Notochord Cells in Intervertebral Disc Development and Degeneration. Journal of Developmental Biology 4, 3.

Pandey, K.B., and Rizvi, S.I. (2011). Biomarkers of oxidative stress in red blood cells. Biomedical Papers of the Medical Faculty of Palacky University in Olomouc 155.

Panebianco, C.J., Dave, A., Charytonowicz, D., Sebra, R., and latridis, J.C. (2021). Single-cell RNA-sequencing atlas of bovine caudal intervertebral discs: Discovery of heterogeneous cell populations with distinct roles in homeostasis. The FASEB Journal 35, e21919. https://doi.org/10.1096/fj.202101149R.

Pratsinis, H., and Kletsas, D. (2007). PDGF, bFGF and IGF-I stimulate the proliferation of intervertebral disc cells in vitro via the activation of the ERK and Akt signaling pathways. Eur Spine J 16, 1858-1866. 10.1007/s00586-007-0408-9.

Qiu, X., Hill, A., Packer, J., Lin, D., Ma, Y.A., and Trapnell, C. (2017a). Single-cell mRNA quantification and differential analysis with Census. Nat Methods 14, 309-315. 10.1038/nmeth.4150.

Qiu, X., Mao, Q., Tang, Y., Wang, L., Chawla, R., Pliner, H., and Trapnell, C. (2017b). Reversed graph embedding resolves complex single-cell developmental trajectories. bioRxiv, 110668. 10.1101/110668. 
Richardson, S.M., Ludwinski, F.E., Gnanalingham, K.K., Atkinson, R.A., Freemont, A.J., and Hoyland, J.A. (2017). Notochordal and nucleus pulposus marker expression is maintained by subpopulations of adult human nucleus pulposus cells through aging and degeneration. Sci Rep 7 , 1501. 10.1038/s41598-017-01567-w.

Risbud, M.V., Schaer, T.P., and Shapiro, I.M. (2010). Toward an understanding of the role of notochordal cells in the adult intervertebral disc: From discord to accord. Developmental Dynamics 239, 2141-2148. https://doi.org/10.1002/dvdy.22350.

Risbud, M.V., Schoepflin, Z.R., Mwale, F., Kandel, R.A., Grad, S., latridis, J.C., Sakai, D., and Hoyland, J.A. (2015). Defining the phenotype of young healthy nucleus pulposus cells: Recommendations of the Spine Research Interest Group at the 2014 annual ORS meeting. Journal of Orthopaedic Research 33, 283-293. https://doi.org/10.1002/jor.22789.

Risbud, M.V., and Shapiro, I.M. (2011). Notochordal cells in the adult intervertebral disc: new perspective on an old question. Critical reviews in eukaryotic gene expression 21, 29-41. 10.1615/critreveukargeneexpr.v21.i1.30.

Rodrigues-Pinto, R., Ward, L., Humphreys, M., Zeef, L.A.H., Berry, A., Hanley, K.P., Hanley, N., Richardson, S.M., and Hoyland, J.A. (2018). Human notochordal cell transcriptome unveils potential regulators of cell function in the developing intervertebral disc. Sci Rep 8, 12866. 10.1038/s41598-018-31172-4.

Satija, R., Farrell, J.A., Gennert, D., Schier, A.F., and Regev, A. (2015). Spatial reconstruction of single-cell gene expression data. Nature Biotechnology 33, 495-502. 10.1038/nbt.3192.

Schindelin, J., Arganda-Carreras, I., Frise, E., Kaynig, V., Longair, M., Pietzsch, T., Preibisch, S., Rueden, C., Saalfeld, S., Schmid, B., et al. (2012). Fiji: an open-source platform for biologicalimage analysis. Nature Methods 9, 676-682. 10.1038/nmeth.2019.

Schneider, C.A., Rasband, W.S., and Eliceiri, K.W. (2012). NIH Image to ImageJ: 25 years of image analysis. Nature Methods 9, 671-675. 10.1038/nmeth.2089. 
Schumann, J., Stanko, K., Schliesser, U., Appelt, C., and Sawitzki, B. (2015). Differences in CD44 Surface Expression Levels and Function Discriminates IL-17 and IFN-y Producing Helper T Cells. PLoS One 10, e0132479-e0132479. 10.1371/journal.pone.0132479.

Schwarzer, A.C., Aprill, C.N., Derby, R., Fortin, J., Kine, G., and Bogduk, N. (1994). The relative contributions of the disc and zygapophyseal joint in chronic low back pain. Spine (Phila Pa 1976) 19, 801-806. 10.1097/00007632-199404000-00013.

Séguin, C.A., Chan, D., Dahia, C.L., and Gazit, Z. (2018). Latest advances in intervertebral disc development and progenitor cells. JOR SPINE 1, e1030. 10.1002/jsp2.1030.

Setty, M., Kiseliovas, V., Levine, J., Gayoso, A., Mazutis, L., and Pe'er, D. (2019). Characterization of cell fate probabilities in single-cell data with Palantir. Nature Biotechnology 37 , 451-460. 10.1038/s41587-019-0068-4.

Sheyn, D., Ben-David, S., Tawackoli, W., Zhou, Z., Salehi, K., Bez, M., De Mel, S., Chan, V., Roth, J., Avalos, P., et al. (2019). Human iPSCs can be differentiated into notochordal cells that reduce intervertebral disc degeneration in a porcine model. Theranostics 9, 7506-7524. 10.7150/thno.34898.

Shi, R., Wang, F., Hong, X., Wang, Y.T., Bao, J.P., Cai, F., and Wu, X.T. (2015). The presence of stem cells in potential stem cell niches of the intervertebral disc region: an in vitro study on rats. Eur Spine J 24, 2411-2424. 10.1007/s00586-015-4168-7.

Sinha, A., Fan, V.B., Ramakrishnan, A.-B., Engelhardt, N., Kennell, J., and Cadigan, K.M. (2021). Repression of Wnt/\&\#x3b2;-catenin signaling by SOX9 and Mastermind-like transcriptional coactivator 2. Science Advances 7, eabe0849. doi:10.1126/sciadv.abe0849.

Sinner, D., Kordich, J.J., Spence, J.R., Opoka, R., Rankin, S., Lin, S.-C.J., Jonatan, D., Zorn, A.M., and Wells, J.M. (2007). Sox17 and Sox4 differentially regulate beta-catenin/T-cell factor activity and proliferation of colon carcinoma cells. Mol Cell Biol 27, 7802-7815. 10.1128/MCB.02179-06.

Stark, R., Grzelak, M., and Hadfield, J. (2019). RNA sequencing: the teenage years. Nature Reviews Genetics 20, 631-656. 10.1038/s41576-019-0150-2. 
Stegner, D., Haining, E.J., and Nieswandt, B. (2014). Targeting Glycoprotein VI and the Immunoreceptor Tyrosine-Based Activation Motif Signaling Pathway. Arteriosclerosis, Thrombosis, and Vascular Biology 34, 1615-1620. doi:10.1161/ATVBAHA.114.303408.

Stuart, T., Butler, A., Hoffman, P., Hafemeister, C., Papalexi, E., Mauck, W.M., III, Hao, Y., Stoeckius, M., Smibert, P., and Satija, R. (2019). Comprehensive Integration of Single-Cell Data. Cell 177, 1888-1902.e1821. 10.1016/j.cell.2019.05.031.

Sun, Z., Liu, B., and Luo, Z.-J. (2020). The Immune Privilege of the Intervertebral Disc: Implications for Intervertebral Disc Degeneration Treatment. Int J Med Sci 17, 685-692. 10.7150/ijms.42238.

Takao, T., and Iwaki, T. (2002). A Comparative Study of Localization of Heat Shock Protein 27 and Heat Shock Protein 72 in the Developmental and Degenerative Intervertebral Discs. Spine 27.

Talamo, F., D'Ambrosio, C., Arena, S., Del Vecchio, P., Ledda, L., Zehender, G., Ferrara, L., and Scaloni, A. (2003). Proteins from bovine tissues and biological fluids: Defining a reference electrophoresis map for liver, kidney, muscle, plasma and red blood cells. PROTEOMICS 3, 440460. https://doi.org/10.1002/pmic.200390059.

Tam, V., Chen, P., Yee, A., Solis, N., Klein, T., Kudelko, M., Sharma, R., Chan, W.C., Overall, C.M., Haglund, L., et al. (2020a). DIPPER: a spatiotemporal proteomics atlas of human intervertebral discs for exploring ageing and degeneration dynamics. bioRxiv, 2020.2007.2011.192948. 10.1101/2020.07.11.192948.

Tam, V., Chen, P., Yee, A., Solis, N., Klein, T., Kudelko, M., Sharma, R., Chan, W.C.W., Overall, C.M., Haglund, L., et al. (2020b). DIPPER, a spatiotemporal proteomics atlas of human intervertebral discs for exploring ageing and degeneration dynamics. elife 9, e64940. 10.7554/eLife.64940.

Trapnell, C., Cacchiarelli, D., Grimsby, J., Pokharel, P., Li, S., Morse, M., Lennon, N.J., Livak, K.J., Mikkelsen, T.S., and Rinn, J.L. (2014). The dynamics and regulators of cell fate decisions are revealed by pseudotemporal ordering of single cells. Nat Biotechnol 32, 381-386. 10.1038/nbt.2859. 
Urs, S., Harrington, A., Liaw, L., and Small, D. (2006). Selective expression of an aP2/Fatty Acid Binding Protein 4-Cre transgene in non-adipogenic tissues during embryonic development. Transgenic Res 15, 647-653. 10.1007/s11248-006-9000-z.

van den Akker, G.G., Koenders, M.I., van de Loo, F.A., van Lent, P.L., Davidson, E.B., and van der Kraan, P.M. (2017). Transcriptional profiling distinguishes inner and outer annulus fibrosus from nucleus pulposus in the bovine intervertebral disc. European Spine Journal 26, 2053-2062.

Vergroesen, P.P.A., Kingma, I., Emanuel, K.S., Hoogendoorn, R.J.W., Welting, T.J., van Royen, B.J., van Dieën, J.H., and Smit, T.H. (2015). Mechanics and biology in intervertebral disc degeneration: a vicious circle. Osteoarthritis and Cartilage 23, 1057-1070. https://doi.org/10.1016/j.joca.2015.03.028.

Wang, J., Huang, Y., Huang, L., Shi, K., Wang, J., Zhu, C., Li, L., Zhang, L., Feng, G., Liu, L., and Song, Y. (2021). Novel biomarkers of intervertebral disc cells and evidence of stem cells in the intervertebral disc. Osteoarthritis and Cartilage 29, 389-401. https://doi.org/10.1016/j.joca.2020.12.005.

Wang, W.L., Abramson, J.H., Ganguly, A., and Rosenberg, A.E. (2008). The surgical pathology of notochordal remnants in adult intervertebral disks: a report of 3 cases. Am J Surg Pathol 32, 1123-1129. 10.1097/PAS.0b013e3181757954.

Wang, Z., Butler, P., Ly, D., Spiotto, M., Koong, A., and Yang, G. (2010). Activation of the Unfolded Protein Response in Wound Healing. Journal of Surgical Research 158, 209. 10.1016/j.jss.2009.11.111.

Yamazaki, Y., Zhao, N., Caulfield, T.R., Liu, C.-C., and Bu, G. (2019). Apolipoprotein E and Alzheimer disease: pathobiology and targeting strategies. Nature Reviews Neurology 15, 501518. 10.1038/s41582-019-0228-7.

Zeng, C.-M., Chang, L.-L., Ying, M.-D., Cao, J., He, Q.-J., Zhu, H., and Yang, B. (2017). AldoKeto Reductase AKR1C1-AKR1C4: Functions, Regulation, and Intervention for Anti-cancer Therapy. Front Pharmacol 8, 119-119. 10.3389/fphar.2017.00119. 
Zhang, D., Jin, L., Reames, D.L., Shen, F.H., Shimer, A.L., and Li, X. (2013). Intervertebral disc degeneration and ectopic bone formation in apolipoprotein E knockout mice. J Orthop Res 31 , 210-217. 10.1002/jor.22216.

Zhang, Y., Zhang, Z., Chen, P., Ma, C.Y., Li, C., Au, T.Y.K., Tam, V., Peng, Y., Wu, R., Cheung, K.M.C., et al. (2020). Directed Differentiation of Notochord-like and Nucleus Pulposus-like Cells Using Human Pluripotent Stem Cells. Cell Reports 30, 2791-2806.e2795. https://doi.org/10.1016/j.celrep.2020.01.100.

Zhou, G., Zheng, Q., Engin, F., Munivez, E., Chen, Y., Sebald, E., Krakow, D., and Lee, B. (2006). Dominance of SOX9 function over RUNX2 during skeletogenesis. Proceedings of the National Academy of Sciences 103, 19004-19009. 10.1073/pnas.0605170103.

Ziegler-Heitbrock, H.W., and Ulevitch, R.J. (1993). CD14: cell surface receptor and differentiation marker. Immunol Today 14, 121-125. 10.1016/0167-5699(93)90212-4.

Andersson, G.B. (1999). Epidemiological features of chronic low-back pain. Lancet 354, 581-585. 10.1016/S0140-6736(99)01312-4.

Aszódi, A., Chan, D., Hunziker, E., Bateman, J.F., and Fässler, R. (1998). Collagen II Is Essential for the Removal of the Notochord and the Formation of Intervertebral Discs. Journal of Cell Biology 143, 1399-1412. 10.1083/jcb.143.5.1399.

Bhattaram, P., Penzo-Méndez, A., Sock, E., Colmenares, C., Kaneko, K.J., Vassilev, A., DePamphilis, M.L., Wegner, M., and Lefebvre, V. (2010). Organogenesis relies on SoxC transcription factors for the survival of neural and mesenchymal progenitors. Nature Communications 1, 9. 10.1038/ncomms1008.

Bi, W., Deng, J.M., Zhang, Z., Behringer, R.R., and de Crombrugghe, B. (1999). Sox9 is required for cartilage formation. Nature Genetics 22, 85-89. 10.1038/8792.

Buckley, C.T., Hoyland, J.A., Fujii, K., Pandit, A., latridis, J.C., and Grad, S. (2018). Critical aspects and challenges for intervertebral disc repair and regeneration-Harnessing advances in tissue engineering. JOR SPINE 1, e1029. 10.1002/jsp2.1029. 
Butler, A., Hoffman, P., Smibert, P., Papalexi, E., and Satija, R. (2018). Integrating single-cell transcriptomic data across different conditions, technologies, and species. Nature Biotechnology 36, 411-420. 10.1038/nbt.4096.

Cai, H., Zou, J., Wang, W., and Yang, A. (2021). BMP2 induces hMSC osteogenesis and matrix remodeling. Mol Med Rep 23, 125. 10.3892/mmr.2020.11764.

Chen, W.K., Yu, X.H., Yang, W., Wang, C., He, W.S., Yan, Y.G., Zhang, J., and Wang, W.J. (2017). IncRNAs: novel players in intervertebral disc degeneration and osteoarthritis. Cell Prolif 50. 10.1111/cpr.12313.

D'Amour, K.A., Agulnick, A.D., Eliazer, S., Kelly, O.G., Kroon, E., and Baetge, E.E. (2005). Efficient differentiation of human embryonic stem cells to definitive endoderm. Nature Biotechnology 23, 1534-1541. 10.1038/nbt1163.

de Schepper, E.I., Damen, J., van Meurs, J.B., Ginai, A.Z., Popham, M., Hofman, A., Koes, B.W., and Bierma-Zeinstra, S.M. (2010). The association between lumbar disc degeneration and low back pain: the influence of age, gender, and individual radiographic features. Spine (Phila $\mathrm{Pa}$ 1976) 35, 531-536. 10.1097/BRS.0b013e3181aa5b33.

Fernandes, L.M., Khan, N.M., Trochez, C.M., Duan, M., Diaz-Hernandez, M.E., Presciutti, S.M., Gibson, G., and Drissi, H. (2020). Single-cell RNA-seq identifies unique transcriptional landscapes of human nucleus pulposus and annulus fibrosus cells. Sci Rep 10, 15263. 10.1038/s41598-020-72261-7.

Frank, J.W., Kerr, M.S., Brooker, A.S., DeMaio, S.E., Maetzel, A., Shannon, H.S., Sullivan, T.J., Norman, R.W., and Wells, R.P. (1996). Disability resulting from occupational low back pain. Part I: What do we know about primary prevention? A review of the scientific evidence on prevention before disability begins. Spine (Phila Pa 1976) 21, 2908-2917. 10.1097/00007632-19961215000024.

Frapin, L., Clouet, J., Delplace, V., Fusellier, M., Guicheux, J., and Le Visage, C. (2019). Lessons learned from intervertebral disc pathophysiology to guide rational design of sequential delivery systems for therapeutic biological factors. Advanced Drug Delivery Reviews 149-150, 49-71. https://doi.org/10.1016/j.addr.2019.08.007. 
Gan, Y., He, J., Zhu, J., Xu, Z., Wang, Z., Yan, J., Hu, O., Bai, Z., Chen, L., Xie, Y., et al. (2021). Spatially defined single-cell transcriptional profiling characterizes diverse chondrocyte subtypes and nucleus pulposus progenitors in human intervertebral discs. Bone Research 9, 37. 10.1038/s41413-021-00163-z.

Hao, Y., Hao, S., Andersen-Nissen, E., Mauck, W.M., III, Zheng, S., Butler, A., Lee, M.J., Wilk, A.J., Darby, C., Zager, M., et al. (2021). Integrated analysis of multimodal single-cell data. Cell 184, 3573-3587.e3529. 10.1016/j.cell.2021.04.048.

Hetz, C., Zhang, K., and Kaufman, R.J. (2020). Mechanisms, regulation and functions of the unfolded protein response. Nature Reviews Molecular Cell Biology 21, 421-438. 10.1038/s41580020-0250-z.

Ho, R., Workman, M.J., Mathkar, P., Wu, K., Kim, K.J., O’Rourke, J.G., Kellogg, M., Montel, V., Banuelos, M.G., Arogundade, O.A., et al. (2021). Cross-Comparison of Human iPSC Motor Neuron Models of Familial and Sporadic ALS Reveals Early and Convergent Transcriptomic Disease Signatures. Cell Systems 12, 159-175.e159. 10.1016/j.cels.2020.10.010.

Hunter, C., Matyas, J., and Duncan, N. (2003). The notochordal cell in the nucleus pulposus: a review in the context of tissue engineering. Tissue engineering 9, 667-677.

Johnson, Z.I., Schoepflin, Z.R., Choi, H., Shapiro, I.M., and Risbud, M.V. (2015). Disc in flames: Roles of TNF- $\alpha$ and IL-1 $\beta$ in intervertebral disc degeneration. European cells \& materials 30, 104117. 10.22203/ecm.v030a08.

Kelly, N.H., Huynh, N.P.T., and Guilak, F. (2020). Single cell RNA-sequencing reveals cellular heterogeneity and trajectories of lineage specification during murine embryonic limb development. Matrix Biology 89, 1-10. https://doi.org/10.1016/j.matbio.2019.12.004.

Knezevic, N.N., Mandalia, S., Raasch, J., Knezevic, I., and Candido, K.D. (2017). Treatment of chronic low back pain-new approaches on the horizon. Journal of pain research 10, 1111.

Kondo, N., Yuasa, T., Shimono, K., Tung, W., Okabe, T., Yasuhara, R., Pacifici, M., Zhang, Y., Iwamoto, M., and Enomoto-Iwamoto, M. (2011). Intervertebral disc development is regulated by Wnt/ß-catenin signaling. Spine 36, E513-E518. 10.1097/BRS.0b013e3181f52cb5. 
Lu, S.-Y., Li, M., and Lin, Y.-L. (2010). Mitf induction by RANKL is critical for osteoclastogenesis. Mol Biol Cell 21, 1763-1771. 10.1091/mbc.e09-07-0584.

Macfarlane, G.J., Thomas, E., Croft, P.R., Papageorgiou, A.C., Jayson, M.I.V., and Silman, A.J. (1999). Predictors of early improvement in low back pain amongst consulters to general practice: the influence of pre-morbid and episode-related factors. Pain 80, 113-119. https://doi.org/10.1016/S0304-3959(98)00209-7.

Marín, Y.E., Seiberg, M., and Lin, C.B. (2009). Aldo-keto reductase 1C subfamily genes in skin are UV-inducible: possible role in keratinocytes survival. Experimental Dermatology 18, 611-618. https://doi.org/10.1111/j.1600-0625.2008.00839.x.

Mathys, H., Davila-Velderrain, J., Peng, Z., Gao, F., Mohammadi, S., Young, J.Z., Menon, M., He, L., Abdurrob, F., Jiang, X., et al. (2019). Single-cell transcriptomic analysis of Alzheimer's disease. Nature 570, 332-337. 10.1038/s41586-019-1195-2.

McCann, M.R., and Séguin, C.A. (2016). Notochord Cells in Intervertebral Disc Development and Degeneration. Journal of Developmental Biology 4, 3.

Pandey, K.B., and Rizvi, S.I. (2011). Biomarkers of oxidative stress in red blood cells. Biomedical Papers of the Medical Faculty of Palacky University in Olomouc 155.

Panebianco, C.J., Dave, A., Charytonowicz, D., Sebra, R., and latridis, J.C. (2021). Single-cell RNA-sequencing atlas of bovine caudal intervertebral discs: Discovery of heterogeneous cell populations with distinct roles in homeostasis. The FASEB Journal 35, e21919. https://doi.org/10.1096/fj.202101149R.

Pratsinis, H., and Kletsas, D. (2007). PDGF, bFGF and IGF-I stimulate the proliferation of intervertebral disc cells in vitro via the activation of the ERK and Akt signaling pathways. Eur Spine J 16, 1858-1866. 10.1007/s00586-007-0408-9.

Qiu, X., Hill, A., Packer, J., Lin, D., Ma, Y.A., and Trapnell, C. (2017a). Single-cell mRNA quantification and differential analysis with Census. Nat Methods 14, 309-315. 10.1038/nmeth.4150. 
Qiu, X., Mao, Q., Tang, Y., Wang, L., Chawla, R., Pliner, H., and Trapnell, C. (2017b). Reversed graph embedding resolves complex single-cell developmental trajectories. bioRxiv, 110668. 10.1101/110668.

Richardson, S.M., Ludwinski, F.E., Gnanalingham, K.K., Atkinson, R.A., Freemont, A.J., and Hoyland, J.A. (2017). Notochordal and nucleus pulposus marker expression is maintained by subpopulations of adult human nucleus pulposus cells through aging and degeneration. Sci Rep 7, 1501. 10.1038/s41598-017-01567-w.

Risbud, M.V., Schaer, T.P., and Shapiro, I.M. (2010). Toward an understanding of the role of notochordal cells in the adult intervertebral disc: From discord to accord. Developmental Dynamics 239, 2141-2148. https://doi.org/10.1002/dvdy.22350.

Risbud, M.V., Schoepflin, Z.R., Mwale, F., Kandel, R.A., Grad, S., latridis, J.C., Sakai, D., and Hoyland, J.A. (2015). Defining the phenotype of young healthy nucleus pulposus cells: Recommendations of the Spine Research Interest Group at the 2014 annual ORS meeting. Journal of Orthopaedic Research 33, 283-293. https://doi.org/10.1002/jor.22789.

Risbud, M.V., and Shapiro, I.M. (2011). Notochordal cells in the adult intervertebral disc: new perspective on an old question. Critical reviews in eukaryotic gene expression 21, 29-41. 10.1615/critreveukargeneexpr.v21.i1.30.

Rodrigues-Pinto, R., Ward, L., Humphreys, M., Zeef, L.A.H., Berry, A., Hanley, K.P., Hanley, N., Richardson, S.M., and Hoyland, J.A. (2018). Human notochordal cell transcriptome unveils potential regulators of cell function in the developing intervertebral disc. Sci Rep 8, 12866. 10.1038/s41598-018-31172-4.

Satija, R., Farrell, J.A., Gennert, D., Schier, A.F., and Regev, A. (2015). Spatial reconstruction of single-cell gene expression data. Nature Biotechnology 33, 495-502. 10.1038/nbt.3192.

Schindelin, J., Arganda-Carreras, I., Frise, E., Kaynig, V., Longair, M., Pietzsch, T., Preibisch, S., Rueden, C., Saalfeld, S., Schmid, B., et al. (2012). Fiji: an open-source platform for biologicalimage analysis. Nature Methods 9, 676-682. 10.1038/nmeth.2019. 
Schneider, C.A., Rasband, W.S., and Eliceiri, K.W. (2012). NIH Image to ImageJ: 25 years of image analysis. Nature Methods 9, 671-675. 10.1038/nmeth.2089.

Schumann, J., Stanko, K., Schliesser, U., Appelt, C., and Sawitzki, B. (2015). Differences in CD44 Surface Expression Levels and Function Discriminates IL-17 and IFN-ץ Producing Helper T Cells. PLoS One 10, e0132479-e0132479. 10.1371/journal.pone.0132479.

Séguin, C.A., Chan, D., Dahia, C.L., and Gazit, Z. (2018). Latest advances in intervertebral disc development and progenitor cells. JOR SPINE 1, e1030. 10.1002/jsp2.1030.

Setty, M., Kiseliovas, V., Levine, J., Gayoso, A., Mazutis, L., and Pe'er, D. (2019). Characterization of cell fate probabilities in single-cell data with Palantir. Nature Biotechnology 37, 451-460. 10.1038/s41587-019-0068-4.

Sheyn, D., Ben-David, S., Tawackoli, W., Zhou, Z., Salehi, K., Bez, M., De Mel, S., Chan, V., Roth, J., Avalos, P., et al. (2019). Human iPSCs can be differentiated into notochordal cells that reduce intervertebral disc degeneration in a porcine model. Theranostics 9, 7506-7524. 10.7150/thno.34898.

Shi, R., Wang, F., Hong, X., Wang, Y.T., Bao, J.P., Cai, F., and Wu, X.T. (2015). The presence of stem cells in potential stem cell niches of the intervertebral disc region: an in vitro study on rats. Eur Spine J 24, 2411-2424. 10.1007/s00586-015-4168-7.

Sinha, A., Fan, V.B., Ramakrishnan, A.-B., Engelhardt, N., Kennell, J., and Cadigan, K.M. (2021). Repression of Wnt/\&\#x3b2;-catenin signaling by SOX9 and Mastermind-like transcriptional coactivator 2. Science Advances 7, eabe0849. doi:10.1126/sciadv.abe0849.

Sinner, D., Kordich, J.J., Spence, J.R., Opoka, R., Rankin, S., Lin, S.-C.J., Jonatan, D., Zorn, A.M., and Wells, J.M. (2007). Sox17 and Sox4 differentially regulate beta-catenin/T-cell factor activity and proliferation of colon carcinoma cells. Mol Cell Biol 27, 7802-7815. 10.1128/MCB.02179-06.

Stark, R., Grzelak, M., and Hadfield, J. (2019). RNA sequencing: the teenage years. Nature Reviews Genetics 20, 631-656. 10.1038/s41576-019-0150-2. 
Stegner, D., Haining, E.J., and Nieswandt, B. (2014). Targeting Glycoprotein VI and the Immunoreceptor Tyrosine-Based Activation Motif Signaling Pathway. Arteriosclerosis, Thrombosis, and Vascular Biology 34, 1615-1620. doi:10.1161/ATVBAHA.114.303408.

Stuart, T., Butler, A., Hoffman, P., Hafemeister, C., Papalexi, E., Mauck, W.M., III, Hao, Y., Stoeckius, M., Smibert, P., and Satija, R. (2019). Comprehensive Integration of Single-Cell Data. Cell 177, 1888-1902.e1821. 10.1016/j.cell.2019.05.031.

Sun, Z., Liu, B., and Luo, Z.-J. (2020). The Immune Privilege of the Intervertebral Disc: Implications for Intervertebral Disc Degeneration Treatment. Int J Med Sci 17, 685-692. 10.7150/ijms.42238.

Takao, T., and Iwaki, T. (2002). A Comparative Study of Localization of Heat Shock Protein 27 and Heat Shock Protein 72 in the Developmental and Degenerative Intervertebral Discs. Spine 27.

Talamo, F., D'Ambrosio, C., Arena, S., Del Vecchio, P., Ledda, L., Zehender, G., Ferrara, L., and Scaloni, A. (2003). Proteins from bovine tissues and biological fluids: Defining a reference electrophoresis map for liver, kidney, muscle, plasma and red blood cells. PROTEOMICS 3, 440460. https://doi.org/10.1002/pmic.200390059.

Tam, V., Chen, P., Yee, A., Solis, N., Klein, T., Kudelko, M., Sharma, R., Chan, W.C., Overall, C.M., Haglund, L., et al. (2020a). DIPPER: a spatiotemporal proteomics atlas of human intervertebral discs for exploring ageing and degeneration dynamics. bioRxiv, 2020.2007.2011.192948. 10.1101/2020.07.11.192948.

Tam, V., Chen, P., Yee, A., Solis, N., Klein, T., Kudelko, M., Sharma, R., Chan, W.C.W., Overall, C.M., Haglund, L., et al. (2020b). DIPPER, a spatiotemporal proteomics atlas of human intervertebral discs for exploring ageing and degeneration dynamics. elife 9, e64940. 10.7554/eLife.64940.

Trapnell, C., Cacchiarelli, D., Grimsby, J., Pokharel, P., Li, S., Morse, M., Lennon, N.J., Livak, K.J., Mikkelsen, T.S., and Rinn, J.L. (2014). The dynamics and regulators of cell fate decisions are revealed by pseudotemporal ordering of single cells. Nat Biotechnol 32, 381-386. 10.1038/nbt.2859. 
Urs, S., Harrington, A., Liaw, L., and Small, D. (2006). Selective expression of an aP2/Fatty Acid Binding Protein 4-Cre transgene in non-adipogenic tissues during embryonic development. Transgenic Res 15, 647-653. 10.1007/s11248-006-9000-z.

van den Akker, G.G., Koenders, M.I., van de Loo, F.A., van Lent, P.L., Davidson, E.B., and van der Kraan, P.M. (2017). Transcriptional profiling distinguishes inner and outer annulus fibrosus from nucleus pulposus in the bovine intervertebral disc. European Spine Journal 26, 2053-2062.

Vergroesen, P.P.A., Kingma, I., Emanuel, K.S., Hoogendoorn, R.J.W., Welting, T.J., van Royen, B.J., van Dieën, J.H., and Smit, T.H. (2015). Mechanics and biology in intervertebral disc degeneration: a vicious circle. Osteoarthritis and Cartilage 23, 1057-1070. https://doi.org/10.1016/j.joca.2015.03.028.

Wang, J., Huang, Y., Huang, L., Shi, K., Wang, J., Zhu, C., Li, L., Zhang, L., Feng, G., Liu, L., and Song, Y. (2021). Novel biomarkers of intervertebral disc cells and evidence of stem cells in the intervertebral disc. Osteoarthritis and Cartilage 29, 389-401. https://doi.org/10.1016/j.joca.2020.12.005.

Wang, W.L., Abramson, J.H., Ganguly, A., and Rosenberg, A.E. (2008). The surgical pathology of notochordal remnants in adult intervertebral disks: a report of 3 cases. Am J Surg Pathol 32, 1123-1129. 10.1097/PAS.0b013e3181757954.

Wang, Z., Butler, P., Ly, D., Spiotto, M., Koong, A., and Yang, G. (2010). Activation of the Unfolded Protein Response in Wound Healing. Journal of Surgical Research 158, 209. 10.1016/j.jss.2009.11.111.

Zeng, C.-M., Chang, L.-L., Ying, M.-D., Cao, J., He, Q.-J., Zhu, H., and Yang, B. (2017). AldoKeto Reductase AKR1C1-AKR1C4: Functions, Regulation, and Intervention for Anti-cancer Therapy. Front Pharmacol 8, 119-119. 10.3389/fphar.2017.00119.

Zhang, D., Jin, L., Reames, D.L., Shen, F.H., Shimer, A.L., and Li, X. (2013). Intervertebral disc degeneration and ectopic bone formation in apolipoprotein E knockout mice. J Orthop Res 31, 210-217. 10.1002/jor.22216. 
Zhou, G., Zheng, Q., Engin, F., Munivez, E., Chen, Y., Sebald, E., Krakow, D., and Lee, B. (2006). Dominance of SOX9 function over RUNX2 during skeletogenesis. Proceedings of the National Academy of Sciences 103, 19004-19009. 10.1073/pnas.0605170103.

Ziegler-Heitbrock, H.W., and Ulevitch, R.J. (1993). CD14: cell surface receptor and differentiation marker. Immunol Today 14, 121-125. 10.1016/0167-5699(93)90212-4. 


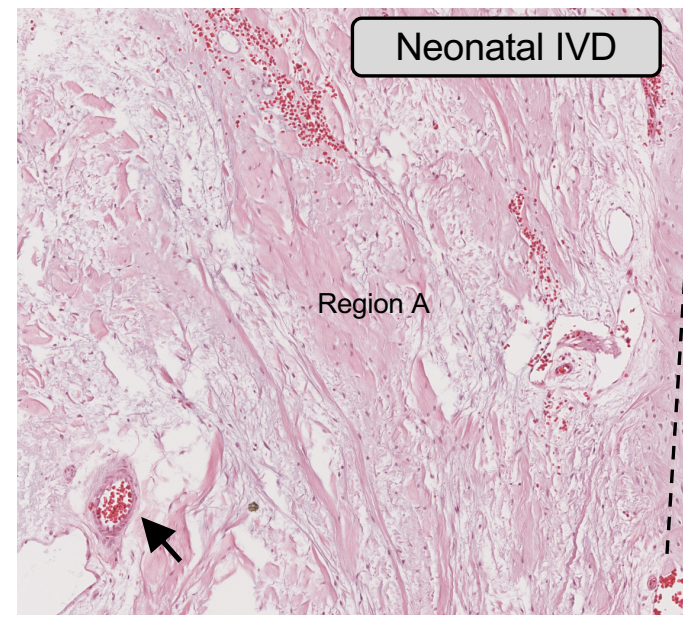

C

Unsupervised Clusters

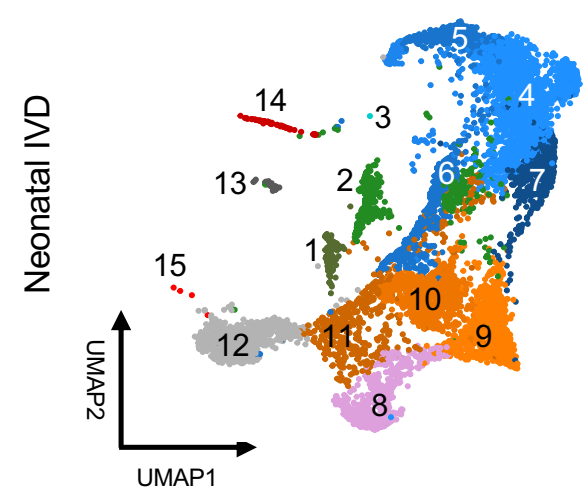

d

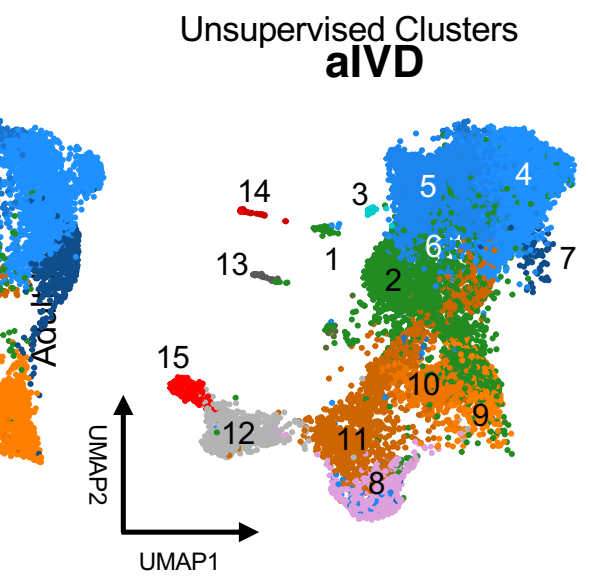

g

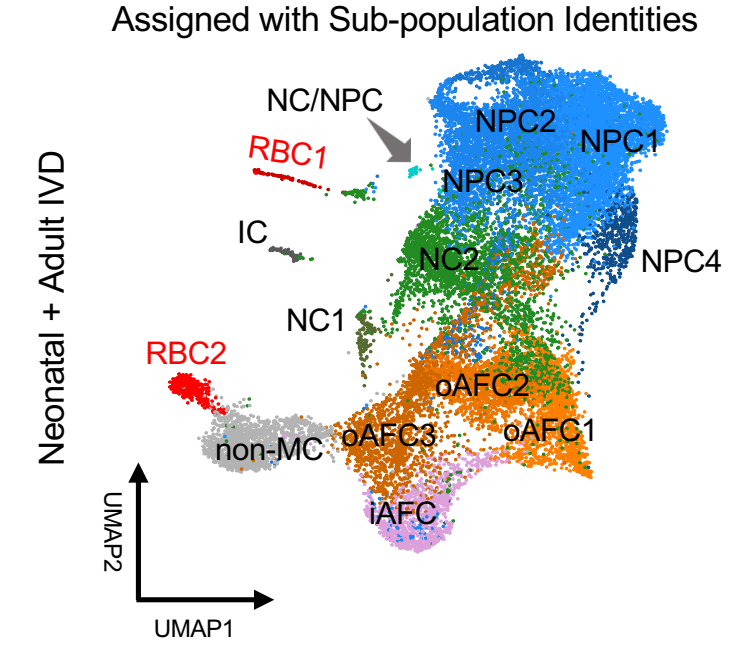

b

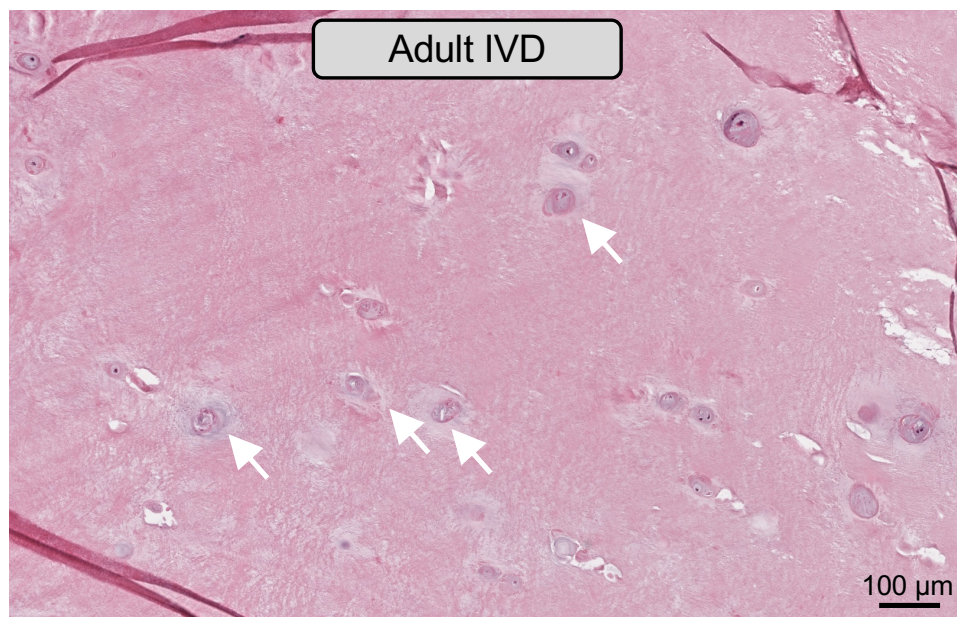

e

IVD-relevant Markers

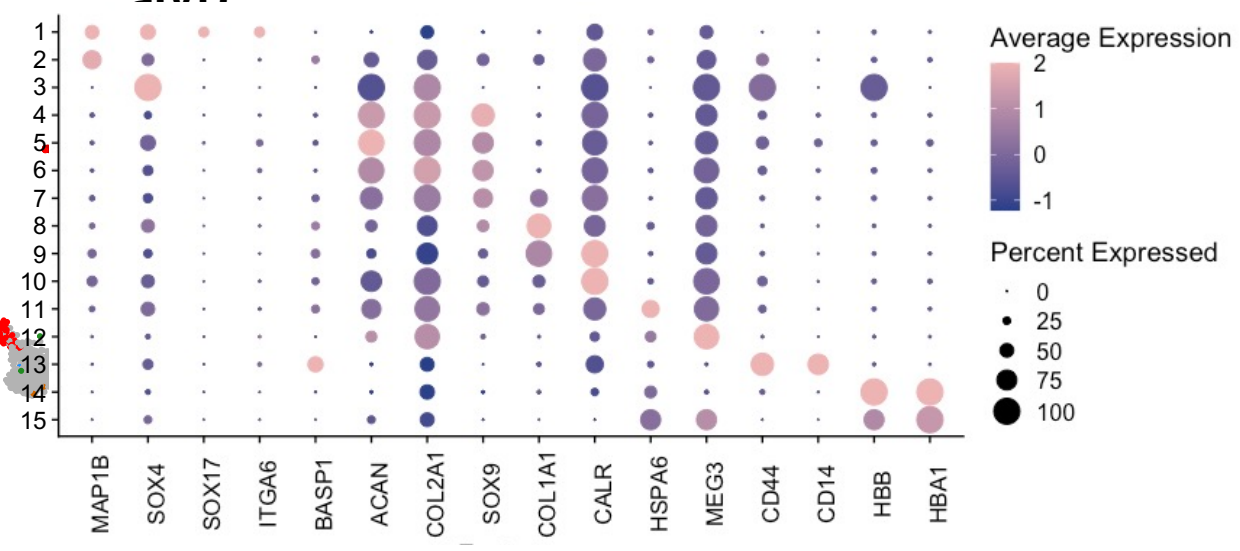

f

IVD-relevant Markers

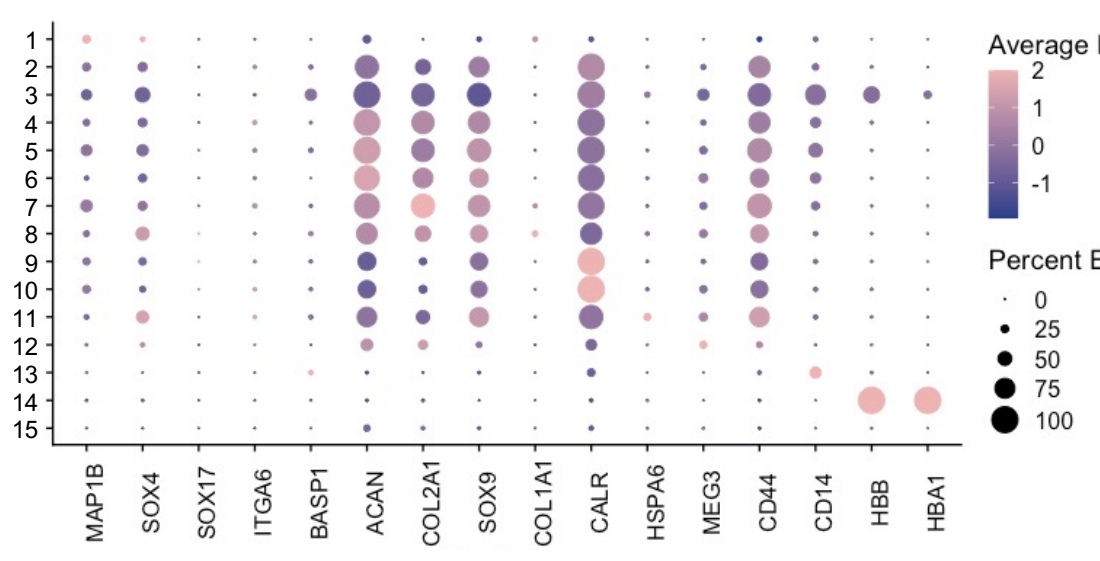

h

Distribution of sub-populations between neonatal and adult IVDs

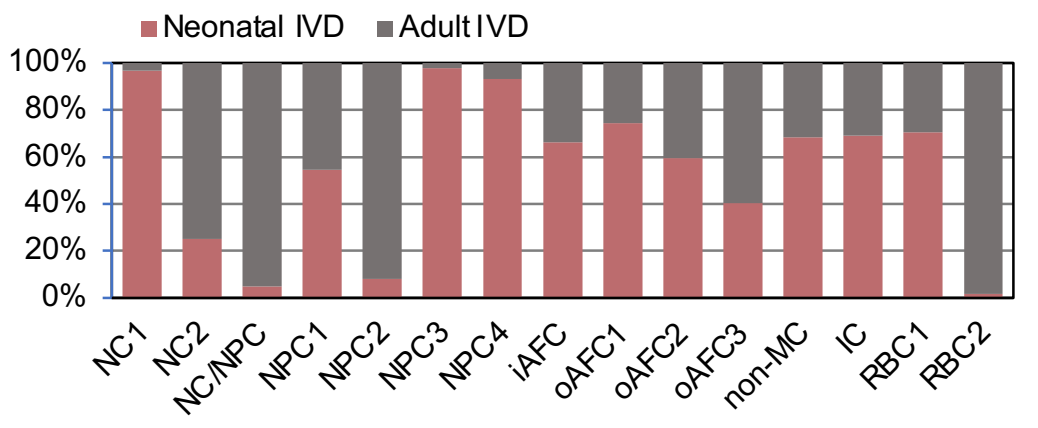



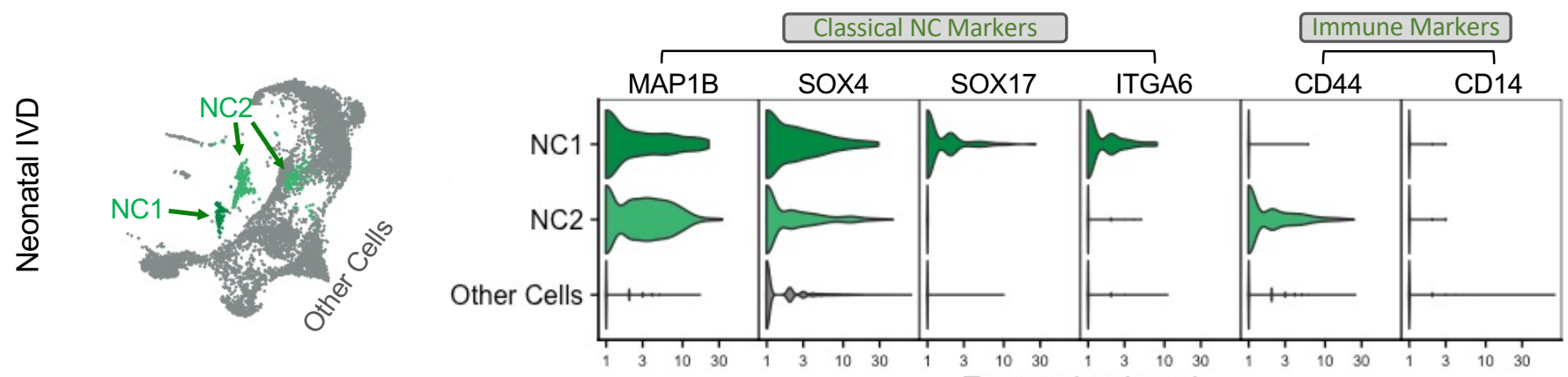

\section{Expression Level}
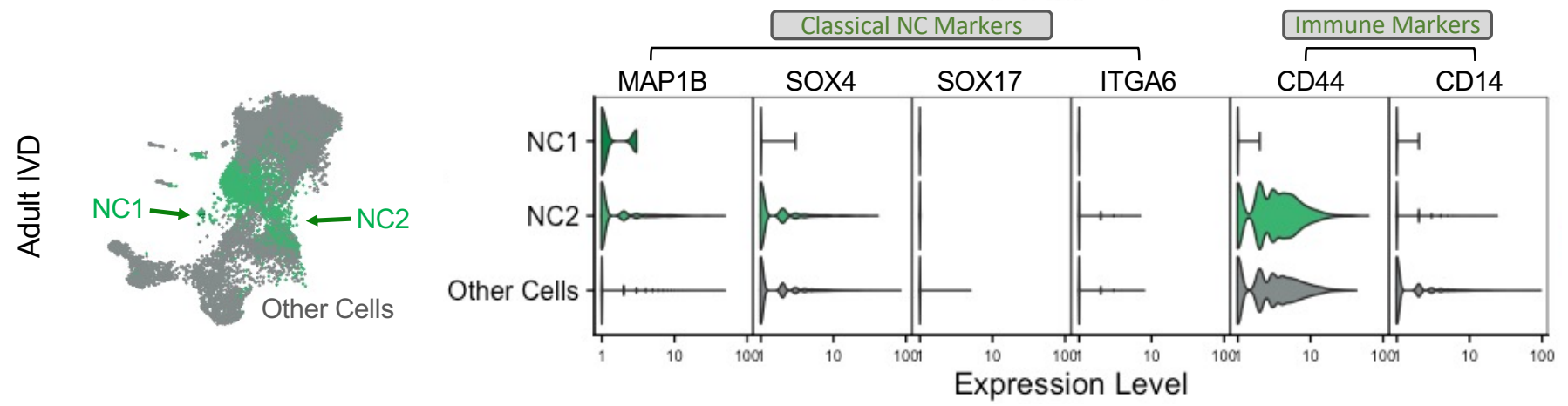

b

Classical NC and Immune Markers

Neonatal IVD
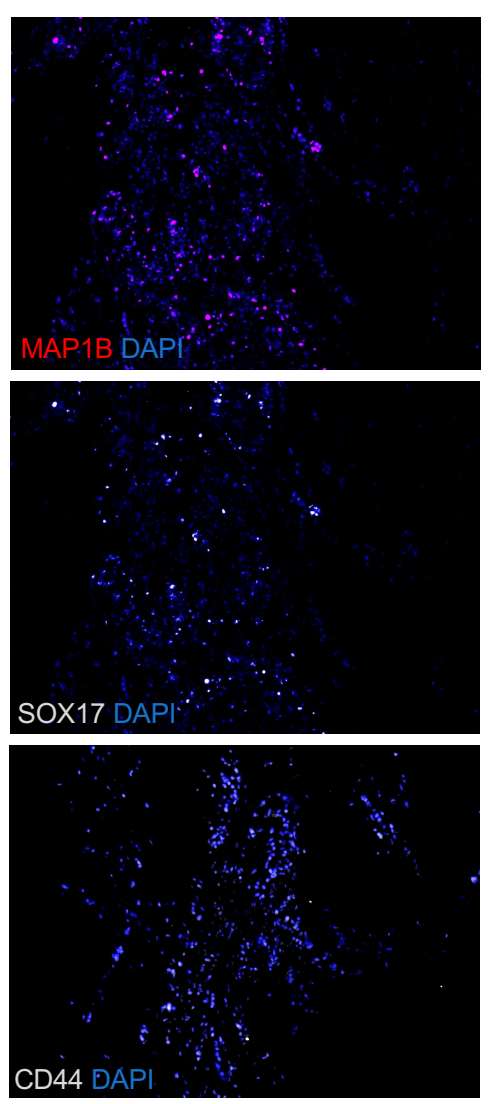

Adult IVD
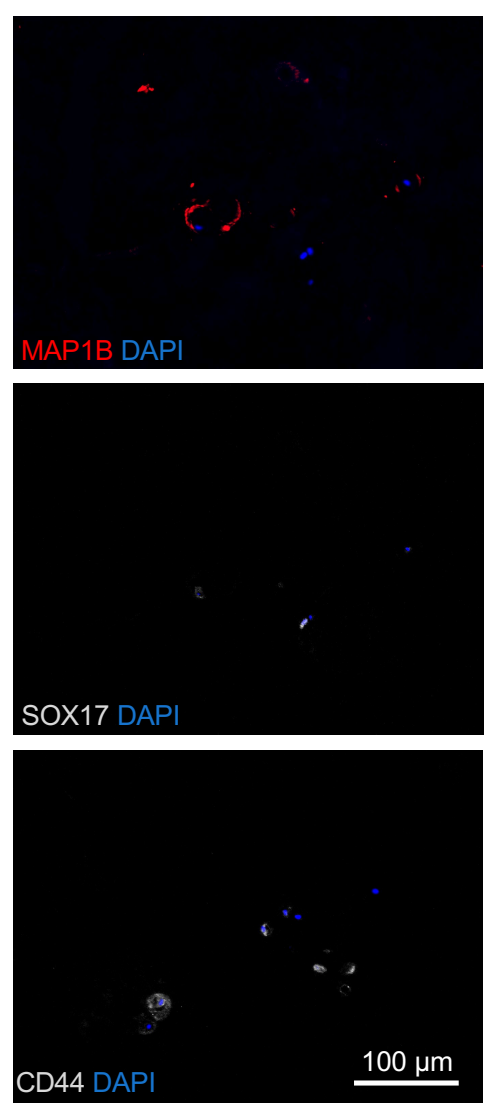

C

Enriched Genes for NC1 \& NC2 Sub-Populations All NC vs. Other Cells
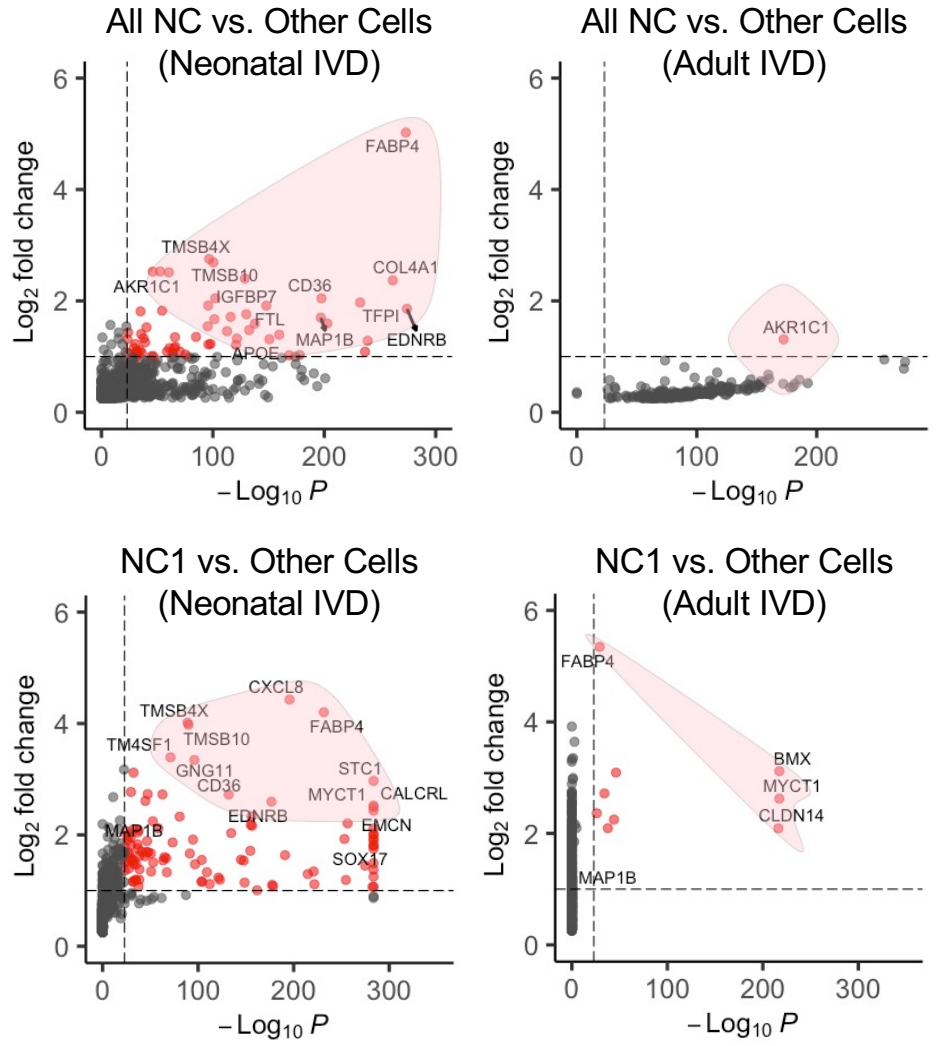

$\mathrm{NC2}$ vs. Other Cells (Neonatal IVD)

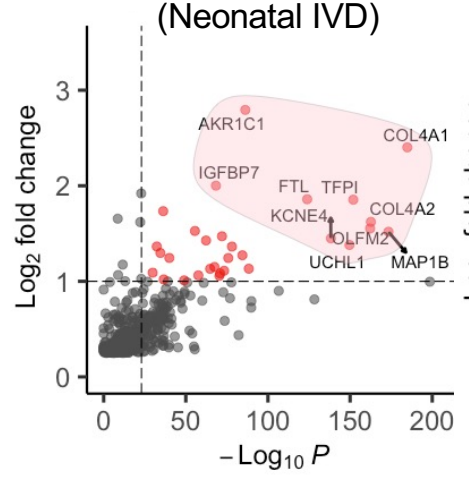

NC2 vs. Other Cells (Adult IVD)

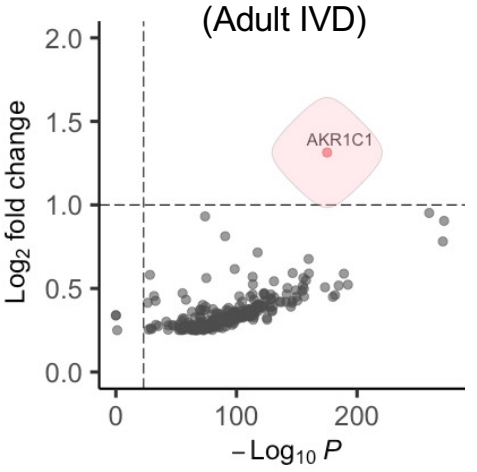




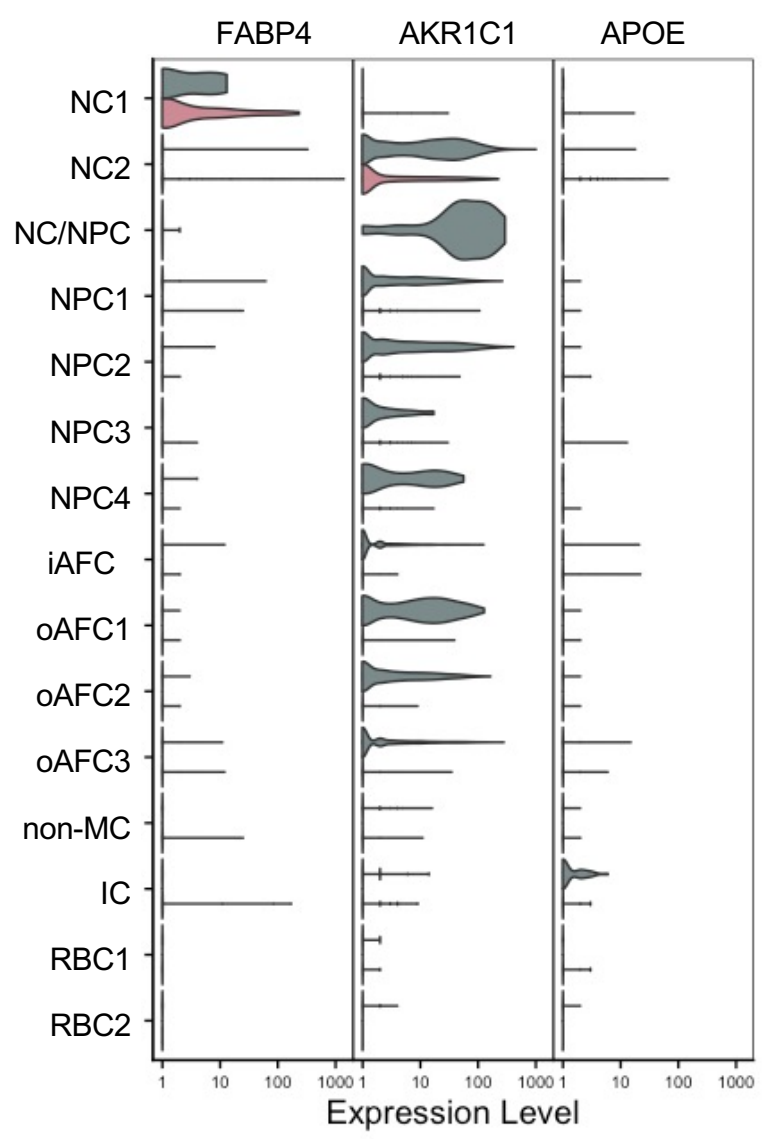

C

FABP4 Marker in NC1 Sub-Populations
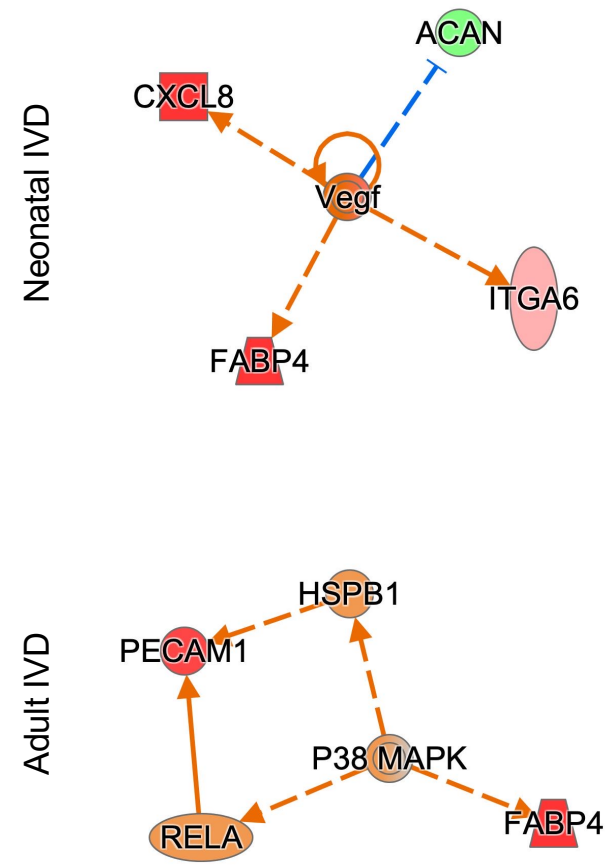

Activation Inhibition Inconclusive
Neonatal IVD Adult IVD
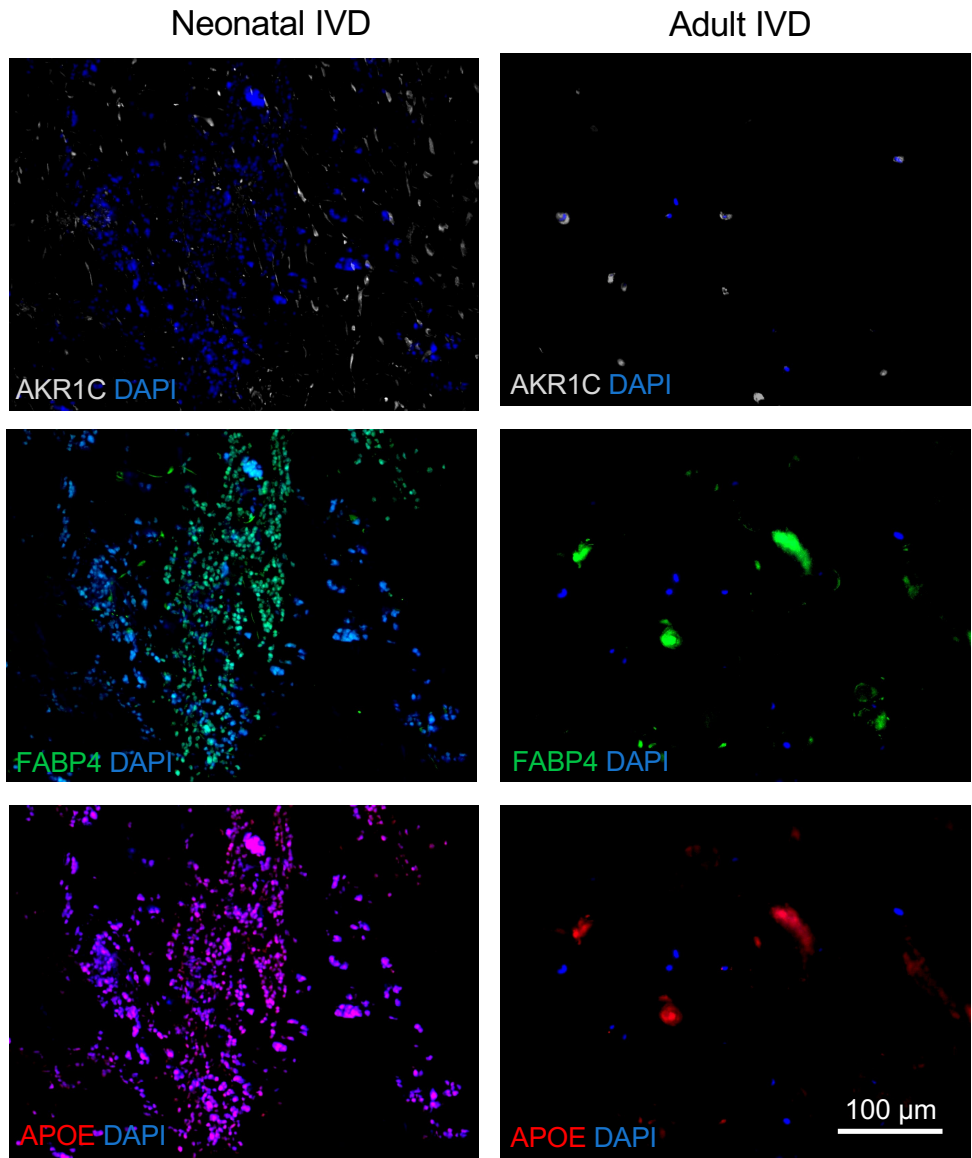

AKR1C1/AKR1C2 Markers in TGFB1 Cascade in Neonatal NC2 Sub-Populations

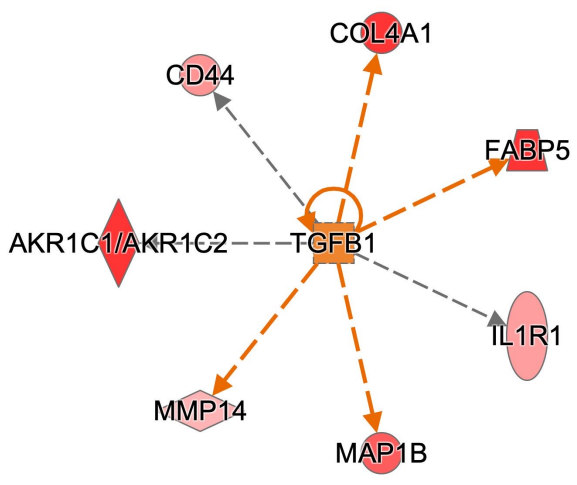

e

Activation Score for AKR1C1/AKR1C2-involved Biological Functions in Neonatal NC1 Sub-Population

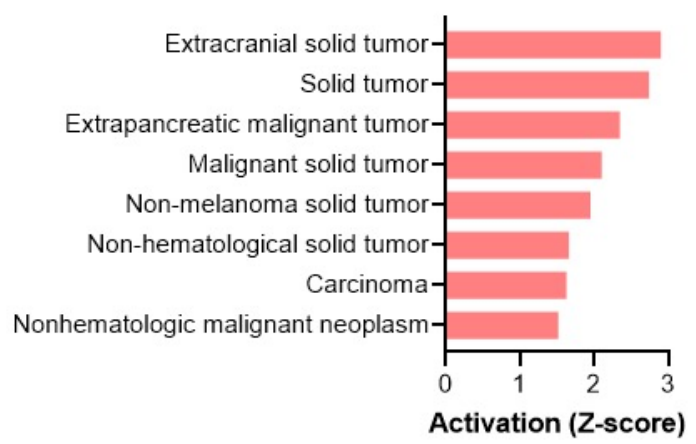



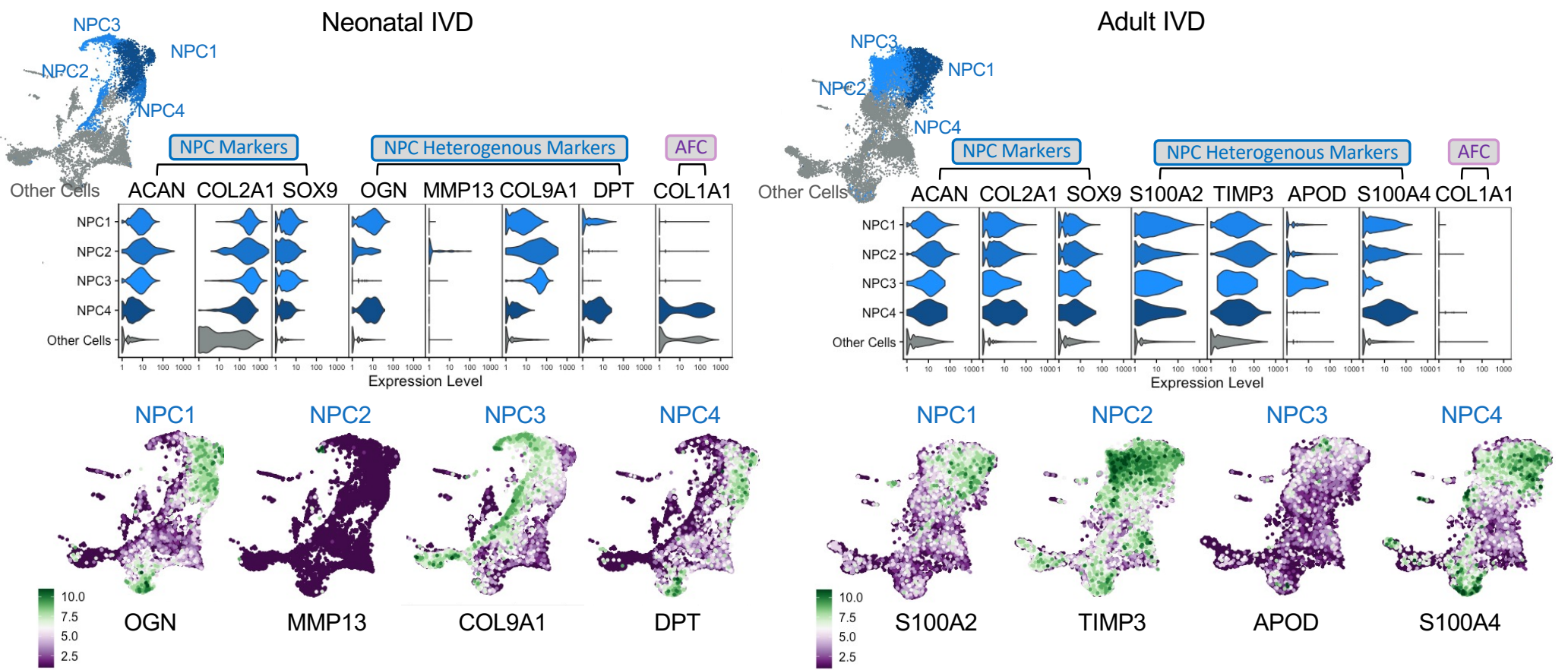

C
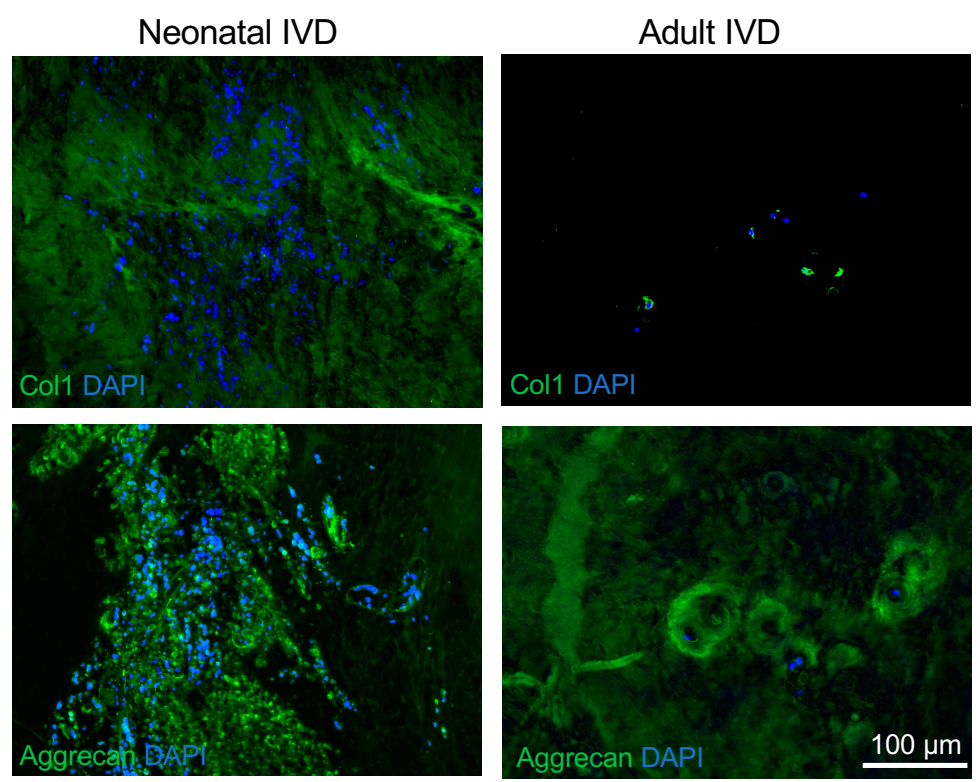

d

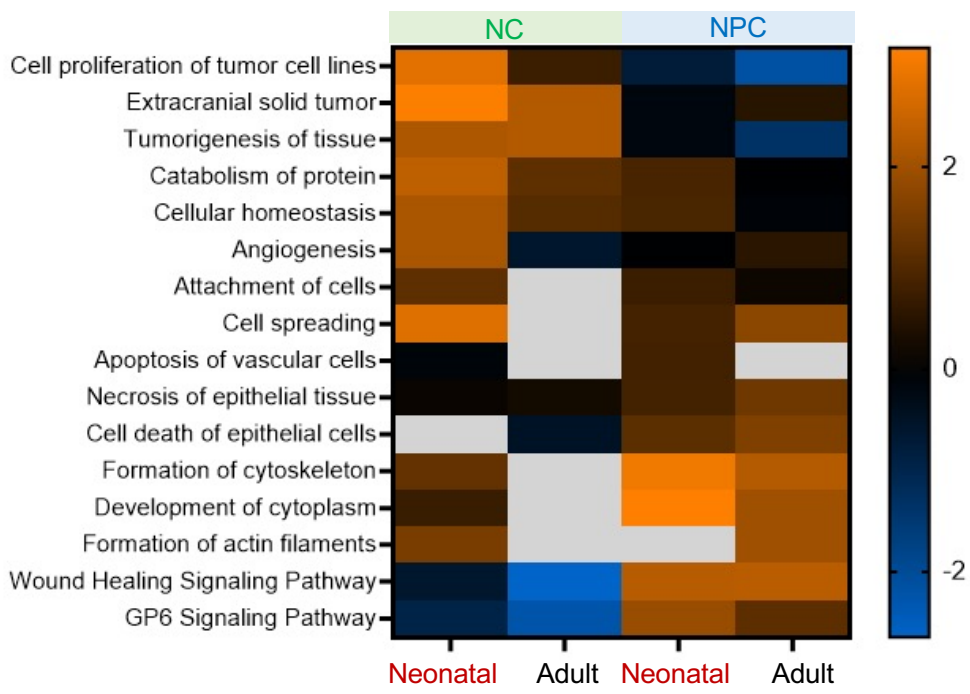

f

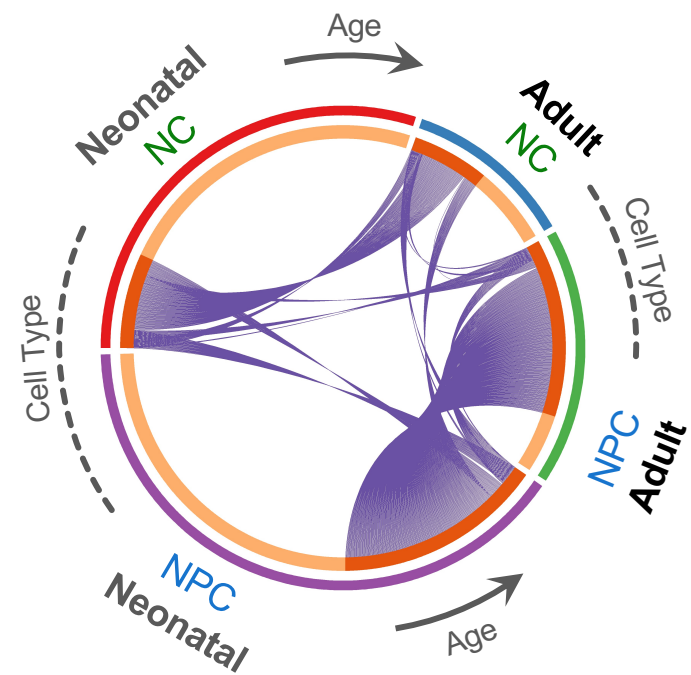

Neonatal

Tumorigenic Inflammatory Cell metabolism

Cell homeostasis

Angiogenesis

Cell adhesion \& spreading

Tissue formation

ECM development

Wound healing

Development of cytoplasm
NC

Tumorigenic

Inflammatory

Cell metabolism

Cell homeostasis

Adult

Epithelial cells apoptosis

Tissue formation

ECM development

Wound healing

Development of cytoplasm 


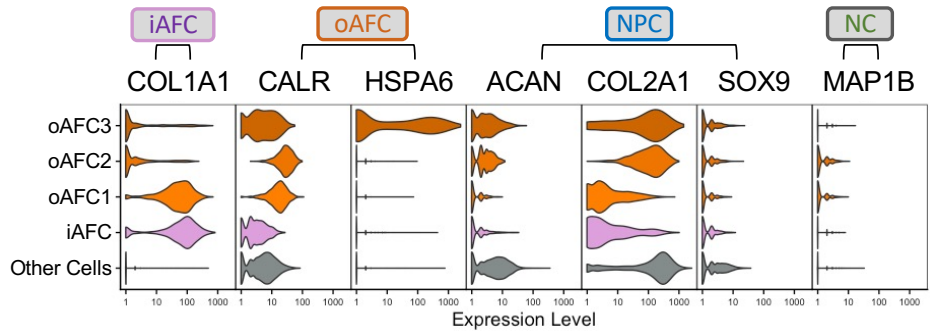

C

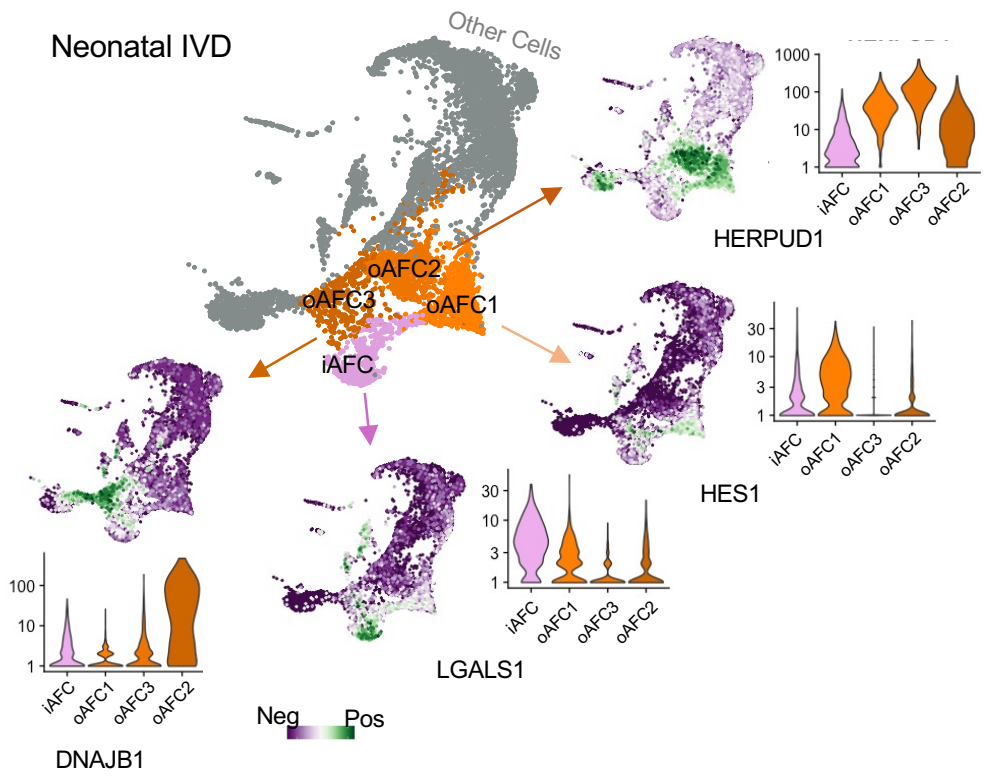

e

Biological Function \& Key Pathway Enrichment for Neonatal Annulus Fibrosus Cell Populations

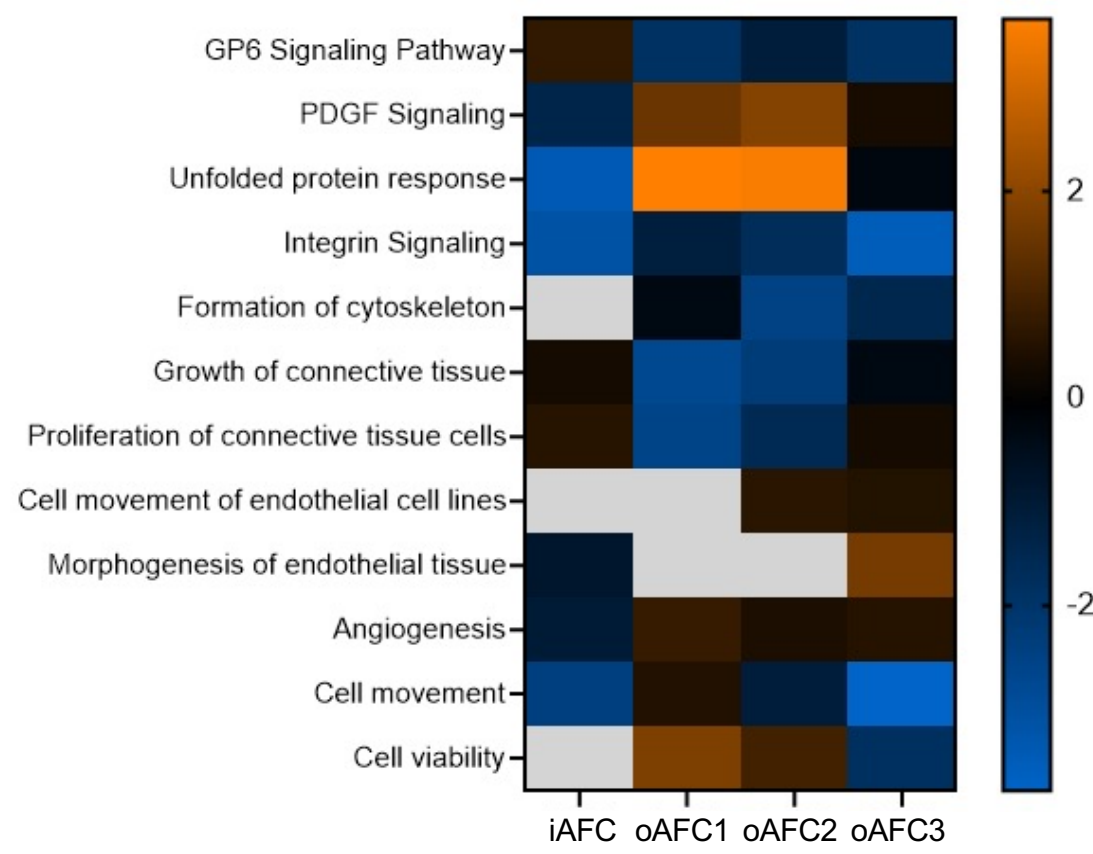

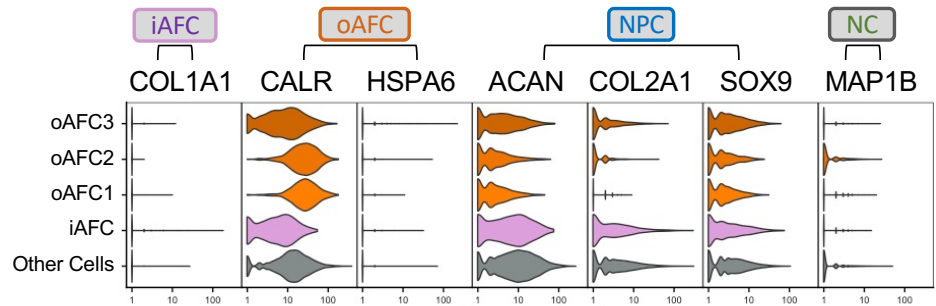

d $\quad$ ECM gradient trend in neonatal IVD

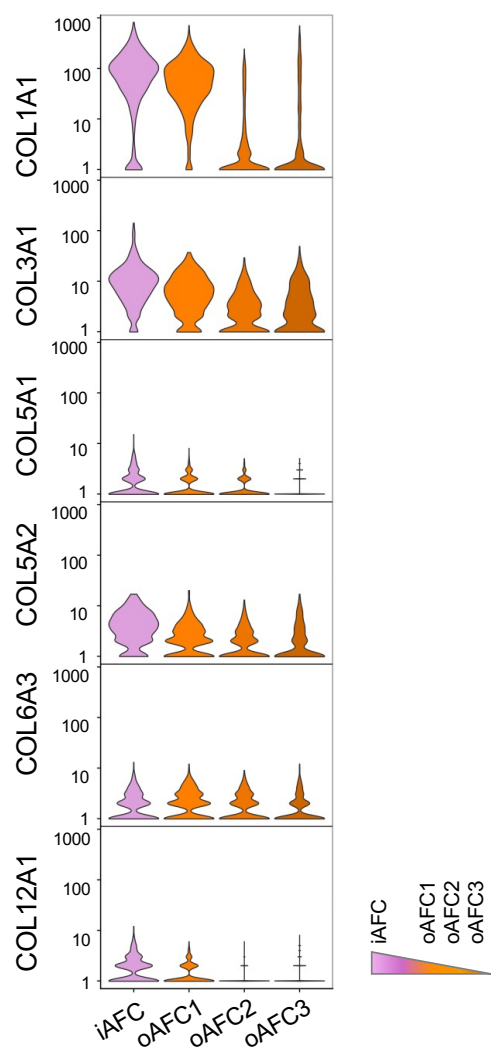

f

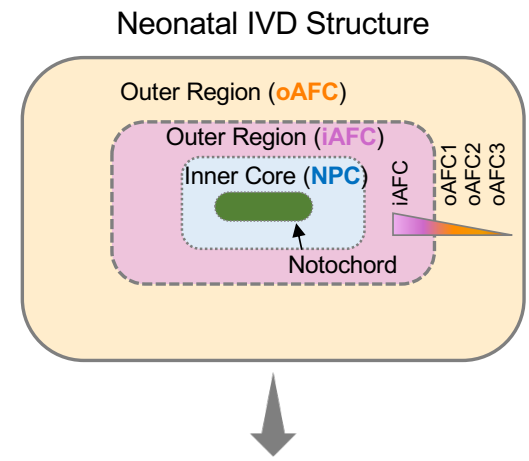

Adult IVD Structure

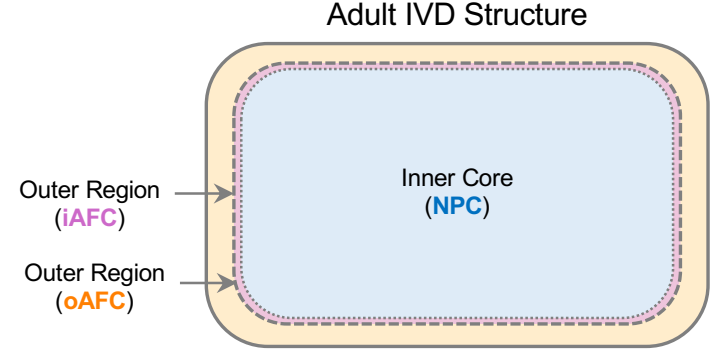




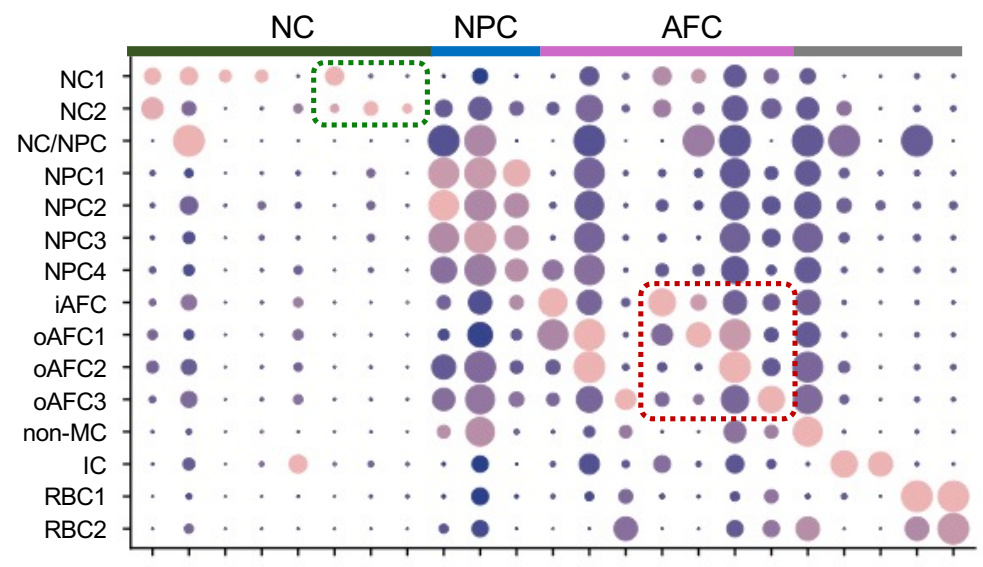

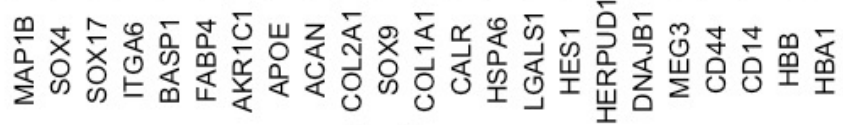

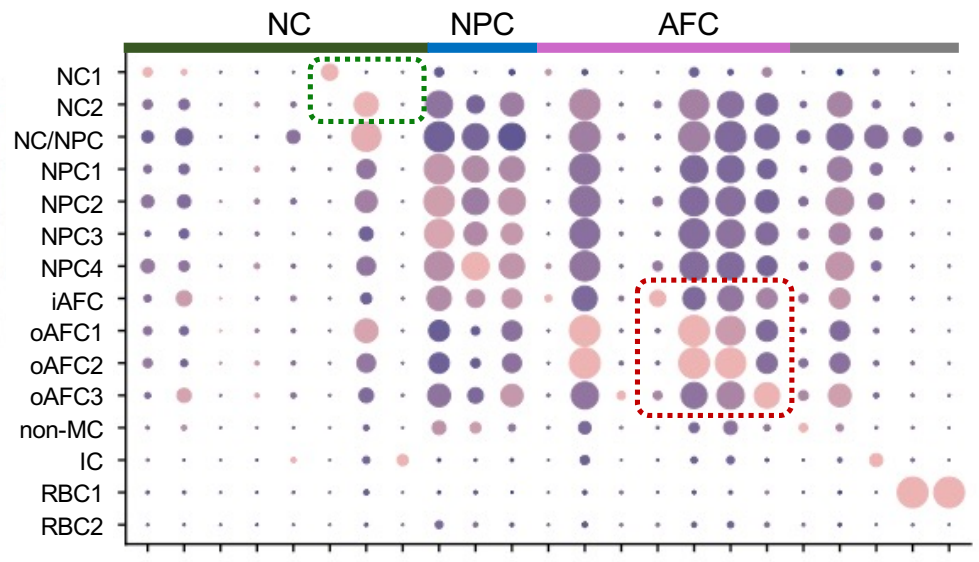

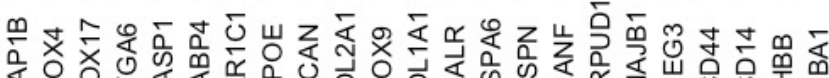

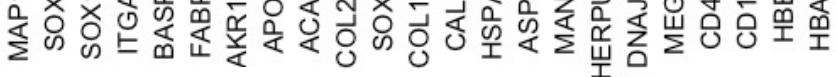

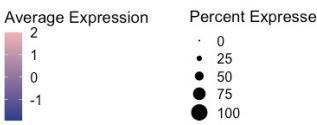

b

Adult IVD

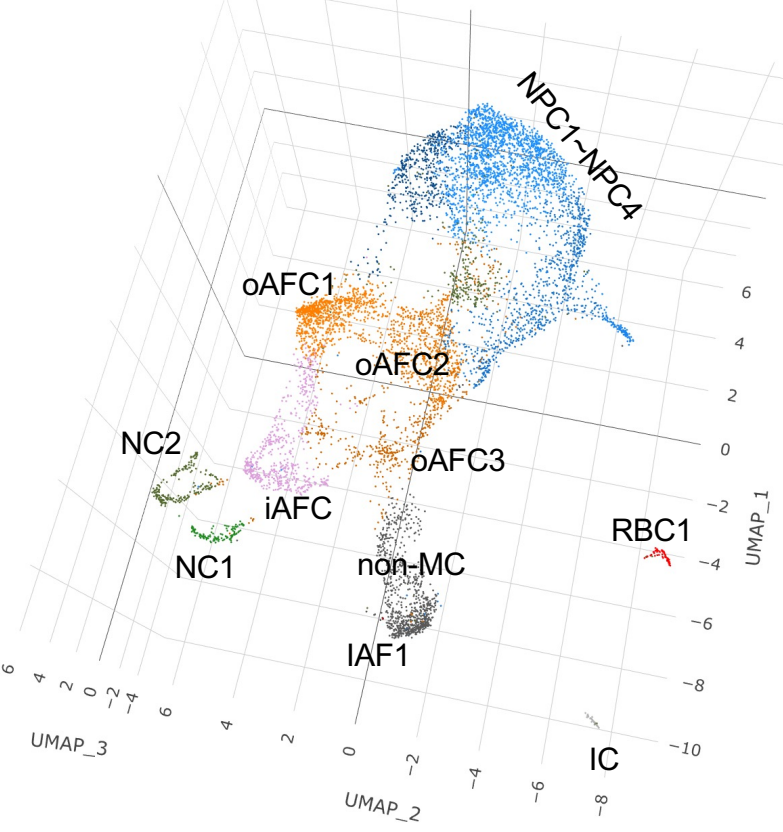

C

d

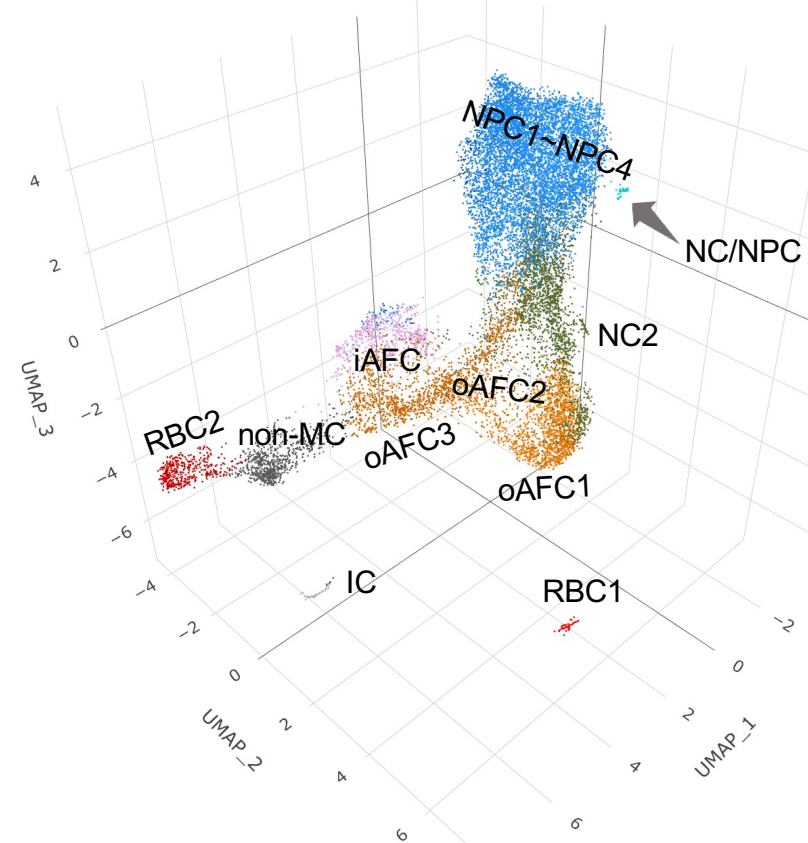

Activation of Wnt/ $\beta$-catenin Pathway

Gene Heatmap for Wnt/ß-catenin Pathway

Wnt/ß-catenin Pathway

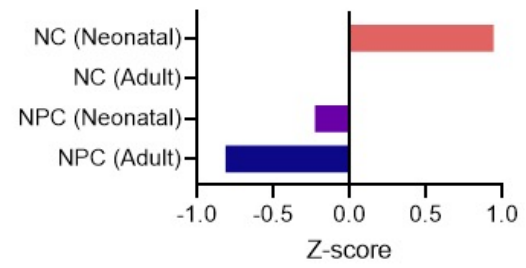

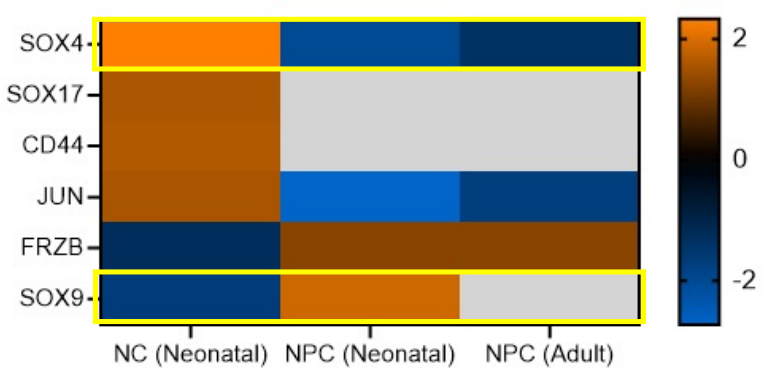




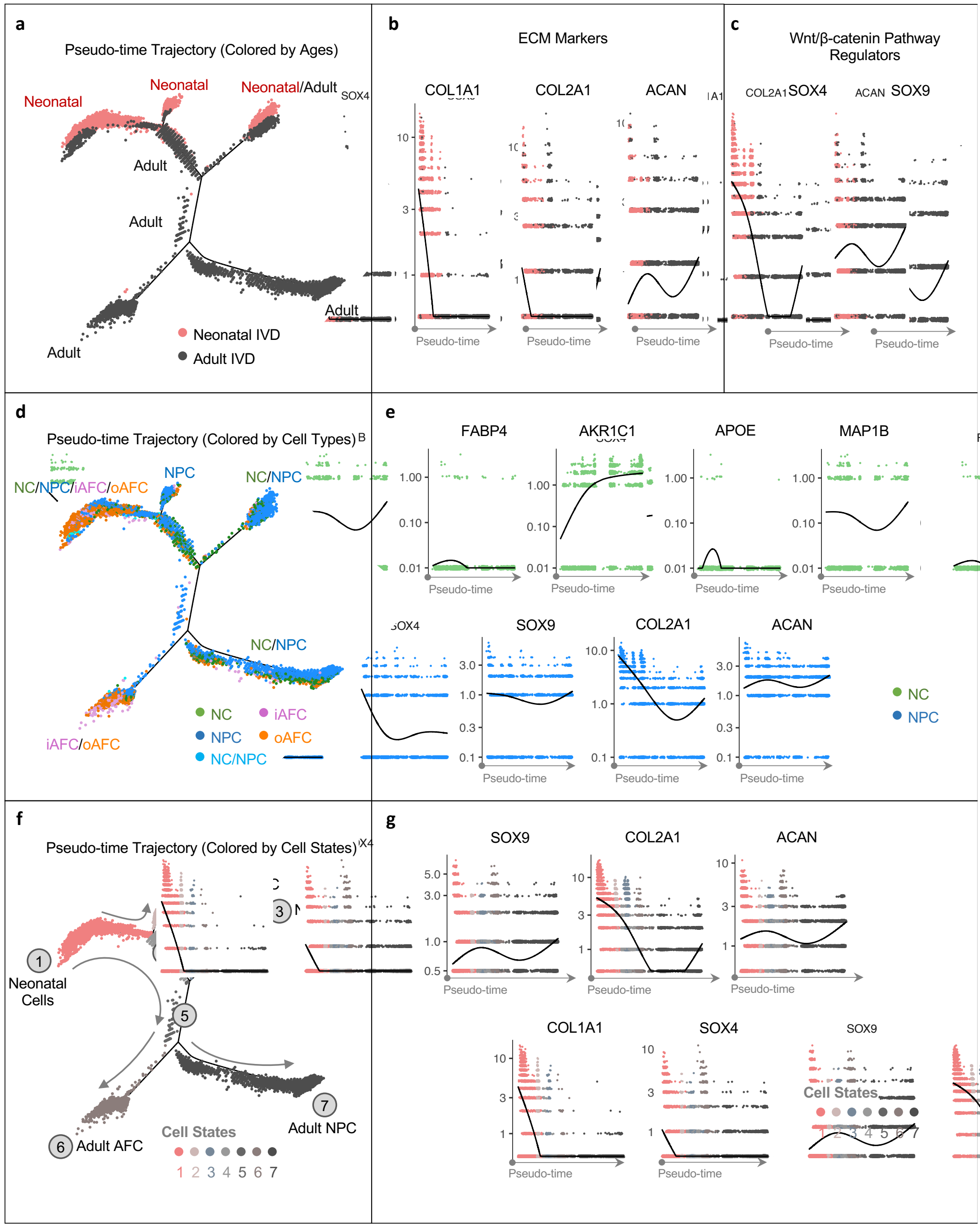

